\title{
Josephson-Anderson Relation and the Classical D'Alembert Paradox
}

\author{
Gregory L. Eyink $\oplus^{1,2}$ \\ ${ }^{1}$ Department of Applied Mathematics \& Statistics, Johns Hopkins University, \\ Baltimore, Maryland 21218, USA \\ ${ }^{2}$ Department of Physics \& Astronomy, Johns Hopkins University, \\ Baltimore, Maryland 21218, USA
}

(Received 28 March 2021; revised 19 July 2021; accepted 30 July 2021; published 10 September 2021)

Generalizing the prior work of P. W. Anderson and E. R. Huggins, we show that a "detailed JosephsonAnderson relation" holds for drag on a finite body held at rest in a classical incompressible fluid flowing with velocity $\mathbf{V}$. The relation asserts an exact equality between the instantaneous power consumption by the drag $-\mathbf{F} \cdot \mathbf{V}$ and the vorticity flux across the potential mass current $-(1 / 2) \int d J \int \epsilon_{i j k} \Sigma_{i j} d \ell_{k}$. Here, $\Sigma_{i j}$ is the flux in the $i$ th coordinate direction of the conserved $j$ th component of vorticity, and the line integrals over $\boldsymbol{\ell}$ are taken along streamlines of the potential-flow solution $\mathbf{u}_{\phi}=\boldsymbol{\nabla} \phi$ of the ideal Euler equation, carrying mass flux $d J=\rho \mathbf{u}_{\phi} \cdot d \mathbf{A}$. Drag and dissipation are thus associated with the motion of vorticity relative to this background ideal potential flow solving Euler's equation. The results generalize the theories of M. J. Lighthill for flow past a body and, in particular, the steady-state relation $(1 / 2) \epsilon_{i j k}\left\langle\Sigma_{j k}\right\rangle=\partial_{i}\langle h\rangle$, where $h=p+(1 / 2)|\mathbf{u}|^{2}$ is the generalized enthalpy or total pressure, extends Lighthill's theory of vorticity generation at solid walls into the interior of the flow. We use these results to explain drag on the body in terms of vortex dynamics, unifying the theories for classical fluids and for quantum superfluids. The results offer a new solution to the "d'Alembert paradox" at infinite Reynolds numbers, provide an explanation for a long-standing puzzle about the experimental conditions required for anomalous turbulent energy dissipation, and imply the necessary and sufficient conditions for turbulent drag reduction.

DOI: 10.1103/PhysRevX.11.031054

\section{INTRODUCTION}

The origin of the Josephson-Anderson relation lies in the work of Josephson on the tunneling of Cooper pairs through normal-superconductor-metal junctions and, in particular, his ac effect [1]. However, the relation assumed its most elegant and powerful statement in the seminal paper of Anderson on flow in superfluid ${ }^{4} \mathrm{He}$ [2]. The relation has had sufficient sustained importance that it garnered two extensive reviews, 30 years [3] and 50 years [4] after Anderson's original work. In its most basic form, it relates the time derivative of the phase $\theta$ of the superfluid macroscopic wave function and the chemical potential $\mu$ as

$$
\hbar \frac{d \theta}{d t}=-\left(\mu+\frac{1}{2} m|\mathbf{u}|^{2}\right)
$$

Published by the American Physical Society under the terms of the Creative Commons Attribution 4.0 International license. Further distribution of this work must maintain attribution to the author(s) and the published article's title, journal citation, and DOI.
Subject Areas: Condensed Matter Physics, Fluid Dynamics, Superfluidity where $\mathbf{u}=\hbar \nabla \theta / m$ is the superfluid velocity. A special significance holds for this relation because topological phase defects exist in superfluids as discrete vortex lines with circulation quantized in units of $\kappa=h / m[5,6]$. The importance of the Josephson-Anderson relation arises from the intimate connection it reveals between force balance and vortex motion. This connection can already be understood by applying a space gradient to Eq. (1.1) which, because $\mathbf{u}$ arises from a multivalued potential, yields the equation of motion

$\frac{d \mathbf{u}}{d t}=\partial_{t} \mathbf{u}+(\mathbf{u} \cdot \boldsymbol{\nabla}) \mathbf{u}=-(1 / m) \boldsymbol{\nabla} \mu+\sum_{V} \mathbf{v}_{V} \times \boldsymbol{\omega}_{V}$,

where the last term on the right is a sum over quantized vortex lines with vorticity $\boldsymbol{\omega}_{V}(\mathbf{x}, t)=\kappa \hat{\mathbf{t}} \delta^{2}\left(\mathbf{r}-\mathbf{r}_{V}(t)\right)$, in which $\hat{\mathbf{t}}$ is a unit vector in the direction of the vorticity, and $\mathbf{r}$ is a two-dimensional coordinate in the transverse plane, with the vortex $V$ located at position $\mathbf{r}_{V}(t)$ and moving with transverse velocity $\mathbf{v}_{V}(t)=d \mathbf{r}_{V}(t) / d t$ [7]. A superfluid would be expected to flow without any applied chemical potential gradient (or pressure gradient, for the incompressible isothermal limit), but Anderson showed that a 


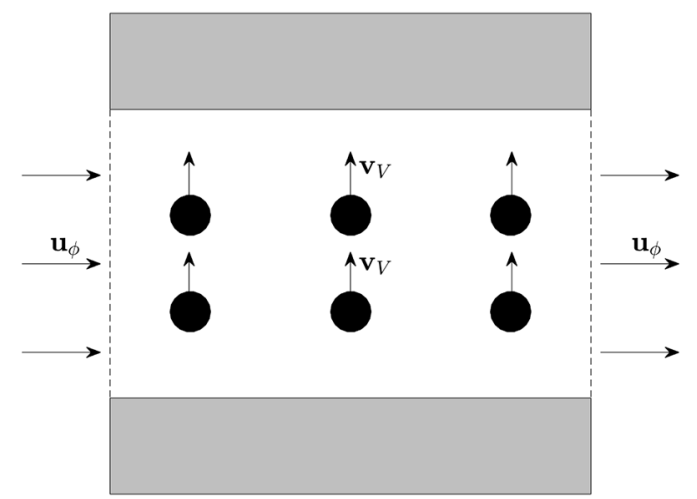

FIG. 1. Dissipative vortex motions in superfluid channel flow according to the Josephson-Anderson relation. Solid black dots denote quantized vortices pointing into the plane and moving across flow lines of the background superfluid velocity $\mathbf{u}_{\phi}$ with a transverse vortex velocity $\mathbf{v}_{V}$, which may arise either from vortexinduced motions or from nonideal effects. When a single vortex crosses the entire channel width, the superfluid phase difference $\Delta \theta$ between outflow and inflow points changes by $2 \pi$, a so-called $2 \pi$ phase-slip event.

drop in chemical potential would occur if there were a time-average flux of quantized vortices [2]. See also Josephson [9] for the corresponding effect of voltage drop in superconductors.

A significant extension of these ideas was obtained subsequently by Huggins [10] whose "detailed Josephson equation" was further able to relate energy dissipation to vortex motion across streamlines of the potential flow $\mathbf{u}_{\phi}$ associated with the superfluid ground state. See also Refs. [4,11,12] for alternative derivations of Huggins's result and Fig. 1 for a concise description. Such vortex motion by " $2 \pi$ phase slips" has been for many years the standard paradigm for energy dissipation in low-temperature superfluids and superconductors $[13,14]$. Furthermore, because Huggins's detailed relation was derived without any sort of averaging, it could be applied to individual flow realizations, and it was found to yield deep insight into otherwise very complicated vortex dynamics. We may quote from the review of Packard, who wrote that

"the equation provides an elegant short cut to certain predictions ... that involve the complex motion of quantized vortices. These same predictions by other methods require a detailed knowledge of vortex motion and involve considerable computational effort" [3].

The reviews [3,4] both analyzed a large number of concrete examples to justify the remarkable efficacy of the detailed Josephson-Anderson relation to understand and predict complex superfluid vortex dynamics.

That classical fluids should satisfy a similar relation was already understood by Anderson, who devoted the Appendix B of his 1966 article to deriving an analogous result for classical hydrodynamics [2]. He stated that he had been unable to find the same result written anywhere in the literature and conjectured that "it was understood by the 'classics' but is of no value in classical hydrodynamics so was never stated" [15]. Most superfluid physicists have followed Anderson in discounting any significance of the Josephson-Anderson relation for classical fluids. As a typical statement, we may quote from the recent review article of Varoquaux:

"This result is of no special importance in classical hydrodynamics because the velocity circulation carried by each vortex, albeit constant, can take any value, while in the superfluid it is directly related to the phase of the macroscopic wave function and quantized" [4].

A notable exception to this trend was Huggins, whose original paper [10] already demonstrated the validity of his "detailed relation" for classical viscous Navier-Stokes solutions and who later wrote a paper applying his results to classical turbulent channel flow [16,17].

Unrecognized by Anderson and the rest of the superfluid community, however, there were already important applications of closely related ideas in classical hydrodynamics. A very early foreshadowing was work on turbulent pipe flow by Taylor [18], who realized that pressure-drop down the pipe implies transverse vortex motion but who did not pursue the connection further. More important was a seminal work of Lighthill [19], who presented a very broad vision of classical fluid mechanics from the perspective of vortex dynamics, encompassing laminar, transitional, and fully turbulent flow. Just three years before Anderson's work on superfluids, Lighthill argued that vorticity flux from solid walls is fundamentally due to tangential pressure gradients at the wall, and he presented many important applications of this principle to classical incompressible fluid dynamics. In fact, his ideas are closely related to those of Huggins [10,16,20,21], which naturally extend Lighthill's concept of vorticity flux at solid walls into the interior of the flow. These connections were previously discussed by us in a paper on turbulent channel flow [22], which further developed Huggins's ideas on that problem and put them in the context of contemporary work on the "attached-eddy hypothesis."

In this paper, we present a new application of the classical Josephson-Anderson relation to flow past a finite solid body and to the problem of the origin of drag. This is in some ways a much more illuminating application of the relation, although it requires some significant modification of the analysis both of Huggins and of Lighthill. In fact, Lighthill in his paper [19] had discussed this same problem in the rest frame of the fluid, appealing there to Kelvin's minimum-energy theorem [23]. We see that this theorem does not hold in the body frame, and this fact crucially enters into the analysis. Kelvin's theorem involves the 
unique potential-flow solution of the inviscid Euler equation satisfying the no-flow-through condition at the body surface, which, according to the famous result of d'Alembert predicts zero drag around a moving body [2426]. In superfluids, this is no "paradox" but is instead a physically observable phenomenon when the body moves at low speeds below a critical velocity. The origin of superfluid drag above this critical velocity was the subject of a study by Frisch et al. [27], which spawned substantial following literature up to the present time, e.g., Refs. [2839]. Our analysis demonstrates a deep similarity between the origin of drag in classical and quantum fluids, with the Josephson-Anderson relation providing the key unifying concept. In both cases, this relation shows that drag and dissipation are associated with motion of vorticity relative to the background ideal potential flow solving Euler's equation. Although we focus mainly on the classical case, we make various remarks as we proceed concerning the differences and similarities to the quantum case.

Our approach applies to classical flows at any Reynolds number but in particular extends to the infinite Reynolds turbulent regime, making a connection of the d'Alembert paradox with the Onsager theory of dissipative Euler solutions [40-42]. That paradox in its original form arose from the demonstration by d'Alembert [24-26] that smooth solutions of ideal Euler equations predict no drag around an obstacle, in apparent contradiction to experience. This puzzle was resolved, in our view, by Saint-Venant's realization of the importance of even a very small viscosity [43], as further elaborated by Prandtl [44] and others [45]. However, this paradox has recently arisen in a new guise, because of two apparently conflicting facts, one empirical and the other mathematical. The empirical fact from laboratory observations is that the dimensionless drag coefficient $C_{D}(\mathrm{Re})$ for flow around a finite object such as a sphere apparently tends to a nonzero constant value as $\operatorname{Re} \rightarrow \infty$ [46]. The mathematical fact is that, under very modest assumptions, the viscous Navier-Stokes solution must tend in the limit $\operatorname{Re} \rightarrow \infty$ to a weak solution of the incompressible Euler equations [47]. The new form of the "paradox" is then how the limiting weak Euler solution can produce a nonvanishing drag. This puzzle is obviously connected with the Onsager theory of turbulence [40-42], which postulates dissipative weak solutions of the Euler equations in order to explain the related empirical observation of "anomalous dissipation," i.e., viscous energy dissipation nonvanishing in the Reynolds-number limit. We argue that the Josephson-Anderson relation and the Onsager theory of the dissipative anomaly complement and mutually illuminate each other.

Our new result can, in fact, resolve a long-standing experimental puzzle [48] regarding the conditions required for an energy-dissipation anomaly in closed-wall-bounded flows versus those required in open flows past obstacles. It is well known from laboratory experiments on closed geometries such as pipe flow [49-52], Taylor-Couette [48], Rayleigh-Bénard [53-55], and the von Kármán flow, or "French washing machine" with rotating disks [48], that energy dissipation, in fact, vanishes slowly with Reynolds number, and there is no strict dissipative anomaly when the bounding walls are hydraulically smooth. To obtain anomalous dissipation in such internal flows, one must instead have rough walls with grains, ridges, or other small features standing perpendicular to the wall, as observed for pipe flow [56-58], Taylor-Couette and von Kármán flows [48], and Rayleigh-Bénard (at least in numerical simulation) [59]. In stark contrast, external flows past obstacles have provided the best experimental evidence for a dissipative anomaly, in flow past grids in wind tunnels $[60,61]$, in jets through orifices in plates [62], and in wakes behind bluff bodies such as cylinders [63] and spheres [46], and in these flows surface roughness is not a requisite for the existence of a dissipative anomaly. Roughness alters the asymptotic value of the dissipative drag in such flows and can even reduce it, e.g., by causing boundary-layer reattachment as in the well-known case of dimples on a golf ball [64], but anomalous dissipation is observed then also with smooth surfaces. As we see, the Josephson-Anderson relation offers an explanation for these diverse experimental observations. See Sec. VA.

In addition, our analysis raises fundamental new questions in the theory of quantum superfluids and, perhaps most importantly, opens up entirely new avenues in the empirical investigation of drag generation and drag reduction for classical incompressible fluids. As we explain, the flow quantities entering in the Josephson-Anderson relation are not only calculable in numerical simulations of the incompressible Navier-Stokes equation but are also measurable in the laboratory by cutting-edge experimental techniques which can, for example, permit observation of the full 3D velocity and vorticity fields with a spatial resolution of 10-60 $\mu \mathrm{m}$ [65] and which can finely resolve flow around individual 1-mm-high roughness elements in a turbulent inner layer [66,67]. Because our analytical results provide for the first time an exact relation between the drag force and the vortex motion, they can be exploited in conjunction with such modern computational and experimental methods to identify quantitatively the fundamental mechanisms of drag in terms of the fluid vorticity dynamics.

A brief summary of the contents of the paper is as follows: Section II concisely reviews the essential ideas of Huggins [10,16,20,21] and Lighthill [19] in order to define some of the basic concepts involved in their theories and to explain the close relationships between them. The longest section of the paper, Sec. III, derives our main result, the detailed Josephson-Anderson relation for flow around a finite body. After a brief explanation of the key differences from the prior work of Lighthill and Huggins and an outline of the important ideas underlying the new 
result, the mathematical details are given in six subsections (Secs. III A-III F). However, a reader who is most interested in concrete applications can skip the derivations and go directly to Sec. IV where flow around a sphere is considered as an illustrative example. The detailed Josephson-Anderson relation is first written in concrete form for the case of a sphere (Sec. IVA), then specific predictions are obtained for the vortex dynamics that must underlie observed drag (Sec. IV B), and finally, comparisons are made with related work on quantum superfluids (Sec. IV C). The concluding section (Sec. V) of the paper discusses general implications and ramifications of the detailed Josephson-Anderson relation, on the d'Alembert paradox and the Onsager theory of turbulence (Sec. VA), on novel empirical investigations made possible into drag mechanisms (Sec. V B), and new theoretical questions raised for both classical and quantum fluid flows (Sec. V C). Finally, two Appendixes present supplemental results, a self-contained derivation of Huggin's detailed relation for channel flow (Appendix A) and a short proof of a relation between vortex momentum and impulse that we require in our derivations (Appendix B).

\section{CONCISE REVIEW OF THEORIES OF HUGGINS AND LIGHTHILL}

Although we are principally concerned with simple fluids described by the incompressible Navier-Stokes equation at constant density $\rho$, the ideas of Huggins apply to an extended system of equations with additional accelerations due to forces both conservative $-\nabla U$ for a potential $U$ and nonconservative $\mathbf{g}$ with $\boldsymbol{\nabla} \times \mathbf{g} \neq 0$ written in the form

$$
\partial_{t} \mathbf{u}=\mathbf{u} \times \boldsymbol{\omega}-\nu \boldsymbol{\nabla} \times \boldsymbol{\omega}-\mathbf{g}-\boldsymbol{\nabla}\left(p+U+\frac{1}{2}|\mathbf{u}|^{2}\right),
$$

where $p=P / \rho$ is kinematic pressure and $\nu=\eta / \rho$ is kinematic viscosity. For example, $U$ could be a gravitational or electrostatic potential, and $\mathbf{g}$ could arise from the stress of a polymer additive. Huggins [16,21] observed that equations of the above class can be rewritten componentwise (with the Einstein summation convention for repeated indices hereafter) as

$$
\partial_{t} u_{i}=\frac{1}{2} \epsilon_{i j k} \Sigma_{j k}-\partial_{i} h
$$

in terms of an antisymmetric tensor

$$
\Sigma_{i j}=u_{i} \omega_{j}-u_{j} \omega_{i}+\nu\left(\frac{\partial \omega_{i}}{\partial x_{j}}-\frac{\partial \omega_{j}}{\partial x_{i}}\right)-\epsilon_{i j k} g_{k},
$$

and a generalized enthalpy or total pressure (static pressure plus dynamic pressure)

$$
h=p+U+\frac{1}{2}|\mathbf{u}|^{2} .
$$

The meaning of the tensor $\boldsymbol{\Sigma}$ is discovered by taking the curl of Eq. (2.1) to obtain the analog of the Helmholtz equation for conservation of vorticity

$$
\partial_{t} \boldsymbol{\omega}+\boldsymbol{\nabla} \cdot \boldsymbol{\Sigma}=\mathbf{0}
$$

with $\Sigma_{i j}$ representing the flux of the $j$ th component of vorticity in the $i$ th coordinate direction. For this reason, we refer to $\boldsymbol{\Sigma}$ as the Huggins vorticity-flux tensor.

The various terms in Eq. (2.3) have transparent physical meanings, with the first representing advective transport, the second transport by vortex stretching or tilting, the third term in parentheses viscous transport, and the final term a flux by the Magnus effect transverse to the applied force. The rewriting of the momentum-conservation equation in the form of Eq. (2.2) is the classical Josephson-Anderson relation in its simplest version, which may be compared directly with quantum relation (1.2). It implies, for example, that for a steady solution or for a suitable time average $\langle\cdot\rangle$ (average over a period for an oscillatory solution or long-time ergodic average for a chaotic solution), the mean gradients of $h$ and the mean vorticity fluxes are exactly related by

$$
\frac{1}{2} \epsilon_{i j k}\left\langle\Sigma_{j k}\right\rangle=\partial_{i}\langle h\rangle
$$

Thus, a mean gradient of $h$ must always be associated with a transverse vorticity flux, and vice versa.

Furthermore, Huggins [10,20] see also Ref. [22], Appendix B) derived a less obvious result, the detailed Josephson relation, in the case of a generalized channel flow, as pictured in Fig. 2. Here the fluid velocity is assumed to be specified on the inflow surface $S_{\text {in }}$, outflow surface $S_{\text {out }}$, and at the channel wall $S_{w}$ as

$$
\left.\mathbf{u}\right|_{S_{\text {in }}}=\mathbf{u}_{\text {in }},\left.\quad \mathbf{u}\right|_{S_{\text {out }}}=\mathbf{u}_{\text {out }},\left.\quad \mathbf{u}\right|_{S_{w}}=\mathbf{0} .
$$

As in the proof of the Kelvin minimum-energy theorem (Ref. [23], Ref. [68] Article 45, Ref. [69] Sec. VI. 2, and Ref. [70] Sec. II. 4. 4), Huggins then introduced the unique incompressible potential flow $\mathbf{u}_{\phi}=\boldsymbol{\nabla} \phi$ satisfying the Neumann boundary conditions

$$
\left.\mathbf{n} \cdot \mathbf{u}_{\phi}\right|_{S_{\mathrm{in}}}=\mathbf{n} \cdot \mathbf{u}_{\mathrm{in}},\left.\quad \mathbf{n} \cdot \mathbf{u}_{\phi}\right|_{S_{\mathrm{out}}}=\mathbf{n} \cdot \mathbf{u}_{\mathrm{out}},\left.\quad \mathbf{n} \cdot \mathbf{u}_{\phi}\right|_{S_{\mathrm{w}}}=\mathbf{0},
$$

and the complementary field $\mathbf{u}_{\omega}=\mathbf{u}-\mathbf{u}_{\phi}$ which represents the velocity due to vorticity. It then easily follows that $\mathbf{u}_{\phi}$ and $\mathbf{u}_{\omega}$ are orthogonal 


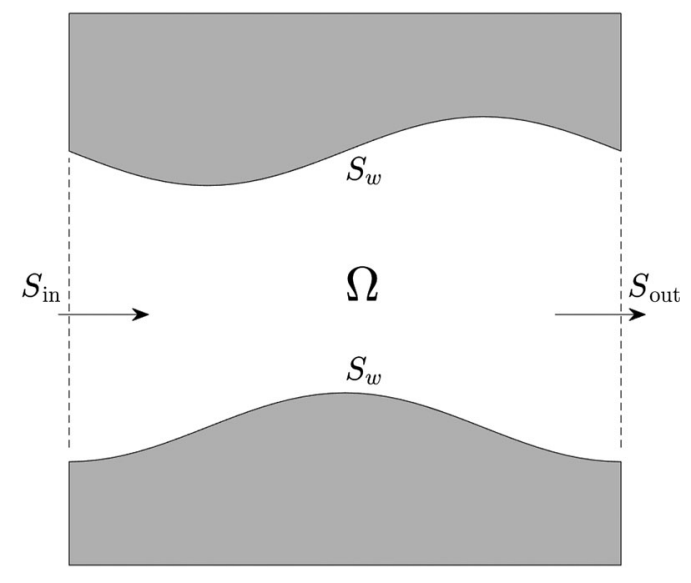

FIG. 2. Flow through a channel $\Omega$ with inflow surface $S_{\text {in }}$, outflow surface $S_{\text {out }}$, and channel walls $S_{w}$.

$$
\int_{\Omega} \mathbf{u}_{\phi} \cdot \mathbf{u}_{\omega} d V=\int_{\Omega} \boldsymbol{\nabla} \cdot\left(\phi \mathbf{u}_{\omega}\right) d V=\int_{\partial \Omega} \phi\left(\mathbf{u}_{\omega} \cdot \hat{\mathbf{n}}\right) d A=0
$$

which is the essence of Kelvin's theorem. Using this orthogonality, Huggins was able to derive equations for energies in the potential flow $E_{\phi}=(\rho / 2) \int_{\Omega}\left|\mathbf{u}_{\phi}\right|^{2} d V$ and in the rotational flow $E_{\omega}=(\rho / 2) \int_{\Omega}\left|\mathbf{u}_{\omega}\right|^{2} d V$ as

$$
\frac{d E_{\phi}}{d t}=\int d J\left(h_{\text {in }}-h_{\text {out }}\right)-\mathcal{T}
$$

and

$$
\frac{d E_{\omega}}{d t}=\mathcal{T}-\int_{\Omega}\left[\eta|\boldsymbol{\omega}|^{2}+\rho \mathbf{u} \cdot \mathbf{g}\right] d V
$$

with

$$
\begin{aligned}
\mathcal{T} & =-\rho \int \mathbf{u}_{\phi} \cdot(\mathbf{u} \times \boldsymbol{\omega}-\nu \boldsymbol{\nabla} \times \boldsymbol{\omega}-\mathbf{g}) d V \\
& =-\int d J \int(\mathbf{u} \times \boldsymbol{\omega}-\nu \boldsymbol{\nabla} \times \boldsymbol{\omega}-\mathbf{g}) \cdot d \ell \\
& =-\frac{1}{2} \int d J \int \epsilon_{i j k} \Sigma_{i j} d \ell_{k} .
\end{aligned}
$$

Here the line integrals are along streamlines of the potential flow, and $d J=\rho \mathbf{u}_{\phi} \cdot d \mathbf{A}$ is the element of mass flux along each streamline, with $\mathcal{T}$ representing transfer of energy from potential to rotational flow by flux of vorticity across mass current. As a consequence of Eq. (2.10), Huggins then obtained the detailed Josephson relation

$$
\mathcal{T}=\int d J\left(h_{\text {in }}^{\prime}-h_{\text {out }}^{\prime}\right)
$$

with $h^{\prime}=h+\dot{\phi}$, which implies an instantaneous equality between the energy-transfer rate and work done by the total pressure field $h^{\prime}$. Note that energy dissipation due to viscosity and other nonpotential forces removes energy only from the rotational motions. See Appendix A for a brief self-contained derivation of Huggins's result.

The velocity decomposition introduced by Huggins is very natural for superfluids, where $\mathbf{u}_{\phi}$ represents the ground-state superfluid velocity and $\mathbf{u}_{\omega}$ the (incompressible) vortical excitations such as vortex rings. As already noted by Landau [71], a quantum superfluid in its zerotemperature ground state may exhibit nontrivial dissipationless superflow, whose velocity $\mathbf{u}_{\phi}$ is the solution of the ideal Euler equation with the appropriate mechanical boundary conditions (applied pressure, moving walls, etc.) The total superfluid velocity $\mathbf{u}$ in Eq. (1.2), on the other hand, contains contributions also from quantized vorticity. It may thus be written in Huggins's form as $\mathbf{u}=\mathbf{u}_{\phi}+\mathbf{u}_{\omega}$, at least in flows where the contribution from the compressible modes (phonons) is negligible. Note, similarly, that $\mathbf{v}_{V}$ in Eq. (1.2) is the total velocity of the vortex $V$, and in general this has contributions both from the background flow $\mathbf{u}_{\phi}$ and also from vortex-induced velocity. One can thus write $\mathbf{v}_{V}=\mathbf{u}_{\phi}+\Delta \mathbf{v}_{V}$, but with only $\Delta \mathbf{v}_{V}$ contributing to effective energy dissipation according to Huggins's detailed relation. Importantly, $\Delta \mathbf{v}_{V}$ may differ as well from $\mathbf{u}_{\omega}$ if there are additional nonideal effects acting on quantized vortices such as mutual friction, which would be roughly analogous in the classical case to viscous diffusion of vorticity. For further discussions of the detailed Josephson-Anderson relation in quantum supefluids, see Refs. [4,11,12].

As we see in Sec. III B, Lighthill [19] introduced the identical decomposition $\mathbf{u}=\mathbf{u}_{\phi}+\mathbf{u}_{\omega}$ in his discussion of flow around a solid body, using quite different arguments. Furthermore, Lighthill recognized that there would be a creation of vorticity at solid walls with a normal flux $\sigma$ related exactly to Huggins's vorticity-flux tensor as

$$
\boldsymbol{\sigma}=\hat{\mathbf{n}} \cdot \boldsymbol{\Sigma}=\hat{\mathbf{n}} \times \nu(\boldsymbol{\nabla} \times \boldsymbol{\omega})+\hat{\mathbf{n}} \times \mathbf{g},
$$

which is now called the Lighthill vorticity source. In fact, Lighthill wrote his source explicitly for a flat wall only, and the general formula above for a curved wall was first proposed by Lyman [72]. There is more than one possible generalization of Lighthill's flat-wall expression to curved walls (e.g., see Refs. [73-76]), but Lyman's proposal is uniquely the one that corresponds to the creation of circulation at the boundary (Ref. [22] Appendix A). Lighthill [19] further realized that vorticity generation at the wall could be related to tangential pressure gradients, as can be seen by substituting the equation of motion (2.1) into Eq. (2.14), to obtain

$$
\boldsymbol{\sigma}=-\hat{\mathbf{n}} \times\left(\boldsymbol{\nabla} h+\partial_{t} \mathbf{u}\right) .
$$


Here we include the term $\partial_{t} \mathbf{u}$, which is nonzero if the wall is accelerating tangential to itself, that was first introduced by Morton [77], who also emphasized the inviscid nature of vorticity generation according to formula (2.15). In common with Josephson and Anderson, Lighthill [19] thus realized also that pressure gradients and transverse vorticity fluxes are inextricably linked.

\section{THE CLASSICAL JOSEPHSON-ANDERSON RELATION FOR A FINITE MOVING BODY}

In this section, we present the derivation of the new Josephson-Anderson relation for flow around a body. A key point unobserved by Lighthill in his discussion of this problem [19] is that the Kelvin minimum-energy theorem holds only in the rest frame of the fluid and not in the body frame. Thus, there is here a nonvanishing "interaction energy" $E_{\text {int }}$ between potential and rotational flow in the body frame, whereas this energy is strictly zero in channel flow according to the result (2.9) exploited by Huggins. Conversely, the energy $E_{\phi}$ of the background potential flow, which in Huggins's relations (2.10) and (2.11) was the source of energy transferred to the rotational motions, instead for flow around a body is conserved (but infinite) by d'Alembert's theorem. As we see below, it is now the interaction energy $E_{\text {int }}$ which is the source for energy transferred to rotational motions, and this transfer $\mathcal{T}$ is described by a formula identical to Huggins's Eq. (2.12) for channel flow. Remarkably, the interaction energy between potential and rotational flow can be directly related by a farfield asymptotic analysis to the total momentum of the rotational wake. Momentum balance then yields the detailed relation between drag on the body and energy transfer to rotational motions, generalizing Huggins's relation (2.13) for channel flow. The far-field analysis is based on a multipole expansion and a careful application of the Lighthill-Morton theory of vorticity generation, which requires extended mathematical discussion.

\section{A. Setup of the problem}

We consider the flow around a finite solid body $B$ with smooth boundary $\partial B$ held at rest in an incompressible fluid that is filling the region $\Omega=\mathbb{R}^{3} \backslash B$ and moving at constant velocity $\mathbf{V}=V \hat{\mathbf{x}}$ upstream of the body and at far distances from it. See Fig. 3. By Galilean invariance, we can equivalently consider the body to be in translational motion with velocity $-V \hat{\mathbf{x}}$ through a fluid at rest and that point of view is sometimes more convenient. In this flow setup, we consider the solution $\mathbf{u}$ of the viscous incompressible Navier-Stokes equation

$$
\partial_{t} \mathbf{u}+\boldsymbol{\nabla} \cdot(\mathbf{u u}+p \mathbf{I}-2 \nu \mathbf{S})=0, \quad \boldsymbol{\nabla} \cdot \mathbf{u}=0
$$

with the boundary conditions
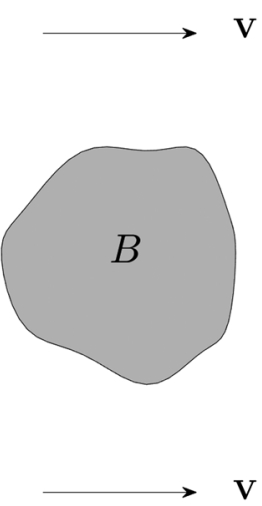

FIG. 3. Flow around a finite body $B$ in an unbounded region $\Omega$ filled with fluid moving at a velocity $\mathbf{V}$ at far distances.

$$
\left.\mathbf{u}\right|_{\partial B}=0, \quad \mathbf{u} \underset{|\mathbf{x}| \rightarrow \infty}{\sim} \mathbf{V} .
$$

Note that in Eq. (3.1) we write the Navier-Stokes equation as a local conservation law for linear momentum, with the total stress tensor (in dyadic notation)

$$
\mathbf{T}=\mathbf{u} \mathbf{u}+p \mathbf{I}-2 \nu \mathbf{S},
$$

where $\mathbf{S}=\frac{1}{2}\left[(\nabla \mathbf{u})+(\nabla \mathbf{u})^{\top}\right]$ is the strain-rate tensor. We assume here that the Navier-Stokes solutions are smooth for all times.

For comparison, we also consider the potential-flow solution of the incompressible Euler equation

$$
\partial_{t} \mathbf{u}_{\phi}+\boldsymbol{\nabla} \cdot\left(\mathbf{u}_{\phi} \mathbf{u}_{\phi}+p_{\phi} \mathbf{I}\right)=\mathbf{0}, \quad \boldsymbol{\nabla} \cdot \mathbf{u}_{\phi}=0
$$

with $\mathbf{u}_{\phi}=\boldsymbol{\nabla} \phi$ given by a velocity potential $\phi$ which satisfies the boundary conditions

$$
\left.\frac{\partial \phi}{\partial n}\right|_{\partial B}=0, \quad \phi \underset{|\mathbf{x}| \rightarrow \infty}{\sim} V x .
$$

Standard theory of potential flow implies that there is a unique smooth solution with potential $\phi$ satisfying the Laplace equation $\triangle \phi=0$ for boundary condition (B.C.) (3.5) and with kinematic pressure given by the Bernoulli equation

$$
\partial_{t} \phi+\frac{1}{2}\left|\mathbf{u}_{\phi}\right|^{2}+p_{\phi}=0 .
$$

Here the pressure $p_{\phi}$ is assumed for convenience to equal the constant value $-\frac{1}{2} V^{2}$ at infinity. This potential flow is, of course, the subject of the famous d'Alembert paradox $[24,26]$ according to which the force exerted by the fluid on the body

$$
\mathbf{F}_{\phi}=-\int_{\partial B} P_{\phi} \hat{\mathbf{n}} d A
$$


has vanishing drag, or $\mathbf{V} \cdot \mathbf{F}_{\phi}=0$. Note that in Eq. (3.7) and hereafter, $\hat{\mathbf{n}}$ denotes the normal at the surface $\partial B$ pointing from the solid body into the fluid.

\section{B. Potential and vortical representation of Navier-Stokes}

Following the approach of Huggins [10] to derive the Josephson-Anderson relation, we introduce the corresponding rotational contributions by the definitions

$$
\mathbf{u}_{\omega}:=\mathbf{u}-\mathbf{u}_{\phi}, \quad p_{\omega}:=p-p_{\phi} .
$$

Although the Navier-Stokes velocity field is thereby decomposed as $\mathbf{u}=\boldsymbol{\nabla} \phi+\boldsymbol{\nabla} \times \boldsymbol{\psi}_{\omega}$ into gradient and curl parts, this is not the familiar Helmholtz decomposition. Recall that the Helmholtz decomposition for a piecewise smooth vector field $\mathbf{v}$ which is zero inside $B$ and smooth in the external flow domain $\Omega$ takes the form

$$
\begin{aligned}
\mathbf{v}(\mathbf{x})= & -\boldsymbol{\nabla}\left(\int_{\Omega} \frac{(\boldsymbol{\nabla} \cdot \mathbf{v})\left(\mathbf{x}^{\prime}\right)}{4 \pi\left|\mathbf{x}-\mathbf{x}^{\prime}\right|} d V^{\prime}+\int_{\partial B} \frac{\hat{\mathbf{n}} \cdot \mathbf{v}\left(\mathbf{x}^{\prime}\right)}{4 \pi\left|\mathbf{x}-\mathbf{x}^{\prime}\right|} d A^{\prime}\right) \\
& +\boldsymbol{\nabla} \times\left(\int_{\Omega} \frac{(\boldsymbol{\nabla} \times \mathbf{v})\left(\mathbf{x}^{\prime}\right)}{4 \pi\left|\mathbf{x}-\mathbf{x}^{\prime}\right|} d V^{\prime}+\int_{\partial B} \frac{\hat{\mathbf{n}} \times \mathbf{v}\left(\mathbf{x}^{\prime}\right)}{4 \pi\left|\mathbf{x}-\mathbf{x}^{\prime}\right|} d A^{\prime}\right) .
\end{aligned}
$$

See Ref. [78] for an engaging discussion of the history of the Helmholtz decomposition and a useful survey of the mathematical literature, and see also Ref. [79] for application to Navier-Stokes solutions outside a solid body. It is important to emphasize that the Helmholtz decomposition cannot be used to obtain uniquely the solenoidal velocity field $\mathbf{u}$ corresponding to a given vorticity field $\omega$ in $\Omega$ without specifying appropriate boundary conditions at $\partial B$ and, furthermore, that arbitrary boundary conditions cannot be imposed. Applied to a Navier-Stokes solution u with B.C. (3.2), the Helmholtz decomposition yields

$$
\mathbf{u}(\mathbf{x}, t)=\boldsymbol{\nabla} \times\left(\int_{\Omega} \frac{\boldsymbol{\omega}\left(\mathbf{x}^{\prime}, t\right)}{4 \pi\left|\mathbf{x}-\mathbf{x}^{\prime}\right|} d V^{\prime}\right) .
$$

As already noted by Lighthill, "there is only a restricted class of vorticity distributions that correspond to real flows satisfying also the no-slip condition" [19].

The velocity field (3.10) is not, however, the only one that yields the vorticity distribution $\boldsymbol{\omega}=\boldsymbol{\nabla} \times \mathbf{u}$. As also noted by Lighthill, "for any given solenoidal distribution of vorticity $\omega$ outside the body (whose motion is again prescribed), one and only one solenoidal velocity field exists, tending to zero at infinity and with zero normal velocity relative to the surface" [19]. In fact, this unique velocity field mentioned by Lighthill for the Navier-Stokes solution $\mathbf{u}$ is exactly the field $\mathbf{u}_{\omega}$ defined in Eq. (3.8), which by its definition satisfies the boundary conditions

$$
\left.\hat{\mathbf{n}} \cdot \mathbf{u}_{\omega}\right|_{\partial B}=0, \quad \hat{\mathbf{n}} \times\left.\mathbf{u}_{\omega}\right|_{\partial B}=-\hat{\mathbf{n}} \times\left.\mathbf{u}_{\phi}\right|_{\partial B}, \quad \mathbf{u}_{\omega|\mathbf{x}| \rightarrow \infty}^{\sim} \mathbf{0},
$$

as a consequence of Eqs. (3.2) and (3.5). The Helmholtz decomposition for this velocity field $\mathbf{u}_{\omega}$ yields the representation

$$
\begin{aligned}
\mathbf{u}_{\boldsymbol{\omega}}(\mathbf{x}, t) & =\boldsymbol{\nabla} \times\left(\int_{\Omega} \frac{\boldsymbol{\omega}\left(\mathbf{x}^{\prime}, t\right)}{4 \pi\left|\mathbf{x}-\mathbf{x}^{\prime}\right|} d V^{\prime}\right. \\
& \left.+\int_{\partial B} \frac{\hat{\mathbf{n}} \times \mathbf{u}_{\omega}\left(\mathbf{x}^{\prime}, t\right)}{4 \pi\left|\mathbf{x}-\mathbf{x}^{\prime}\right|} d A^{\prime}\right),
\end{aligned}
$$

and likewise the Euler solution $\mathbf{u}_{\phi}$ is given by

$$
\mathbf{u}_{\phi}(\mathbf{x}, t)=\boldsymbol{\nabla} \times\left(\int_{\partial B} \frac{\hat{\mathbf{n}} \times \mathbf{u}_{\phi}\left(\mathbf{x}^{\prime}, t\right)}{4 \pi\left|\mathbf{x}-\mathbf{x}^{\prime}\right|} d A^{\prime}\right),
$$

which is an alternative representation of the potential flow as $\mathbf{u}_{\phi}=\boldsymbol{\nabla} \times \boldsymbol{\psi}_{\phi}$ in terms of a vector potential.

The quantities $\hat{\mathbf{n}} \times \mathbf{u}_{\omega}, \hat{\mathbf{n}} \times \mathbf{u}_{\phi}$ in the integral representations (3.12) and (3.13) have a simple physical interpretation as singular vorticity sheets on the surface $\partial B$, equal and opposite to each other. This observation allows us to identify the fields $\mathbf{u}_{\omega}, \mathbf{u}_{\phi}$ introduced by Huggins [10] with corresponding fields that appear in Lagrangian vortex methods for solving the Navier-Stokes equation, which were pioneered by Payne $[80,81]$ and advocated by Lighthill [19]. These methods have since been substantially developed with many alternative schemes proposed. See Ref. [82] for a clear discussion of the application to flow around a solid body, and see Ref. [83] for a recent general review. In the version explained by Lighthill [19], given a vorticity distribution $\omega$ in $\Omega$ at each instant (obtained by advecting, stretching, and diffusing the prior distribution), one uses the Biot-Savart formula to construct the unique solenoidal velocity field which is vanishing at infinity and satisfying no-flow-through conditions at $\partial B$, which is the field $\mathbf{u}_{\omega}$. However, this velocity field does not satisfy the stick B.C. at $\partial B$ on its tangential components and, also, it is not a constant velocity $\mathbf{V}$ at infinity in the body frame. To remedy these defects, one must add a tangential vortex sheet $\hat{\mathbf{n}} \times \mathbf{u}_{\phi}$ at the body surface $\partial B$, so that the resultant velocity field $\mathbf{u}=\mathbf{u}_{\omega}+\mathbf{u}_{\phi}$ satisfies both conditions. More physically, $\hat{\mathbf{n}} \times \mathbf{u}_{\phi}$ is considered as the newly generated vorticity at the surface, which, as pointed out by Lighthill, corresponds to vorticity oriented along equipotential lines of the Euler flow.

We take here, however, a very different point of view. Since the Euler flow $\mathbf{u}_{\phi}$ satisfying the B.C. (3.5) is unique and easily computed, either analytically or numerically, we instead use the definitions (3.8) to introduce a new formulation of incompressible Navier-Stokes which we call the potential-vortical formulation. This formulation can 
be easily obtained by taking the difference of the NavierStokes and Euler equations to obtain an equation of motion for $\mathbf{u}_{\omega}$ of the form

$\partial_{t} \mathbf{u}_{\omega}+\boldsymbol{\nabla} \cdot\left(\mathbf{u}_{\omega} \mathbf{u}_{\omega}+\mathbf{u}_{\phi} \mathbf{u}_{\omega}+\mathbf{u}_{\omega} \mathbf{u}_{\phi}+p_{\omega} \mathbf{I}-2 \nu \mathbf{S}\right)=\mathbf{0}$.

This equation must be solved with the B.C.

$$
\left.\mathbf{u}_{\omega}\right|_{\partial B}=-\left.\mathbf{u}_{\phi}\right|_{\partial B}, \quad \mathbf{u}_{\omega} \underset{|\mathbf{x}| \rightarrow \infty}{\sim} \mathbf{0},
$$

and also the pressure $p_{\omega}$ chosen to enforce the incompressibility condition $\boldsymbol{\nabla} \cdot \mathbf{u}_{\omega}=0$. From the obtained $\mathbf{u}_{\omega}$, the Navier-Stokes solution can then be reconstructed as $\mathbf{u}=\mathbf{u}_{\phi}+\mathbf{u}_{\omega}$. Equation (3.14) can be regarded as expressing the local conservation of the integral $\mathbf{P}_{\omega}=\rho \int_{\Omega} \mathbf{u}_{\omega} d V$, which we call the vortex momentum. Of course, other equivalent forms of Eq. (3.14) can be derived. For example, substituting $\partial_{t} \mathbf{u}_{\phi}=\boldsymbol{\nabla} \dot{\phi}$ and using vector calculus identities yields

$$
\begin{aligned}
\partial_{t} \mathbf{u}_{\omega} & =\mathbf{u} \times \boldsymbol{\omega}-\nu \boldsymbol{\nabla} \times \boldsymbol{\omega}+\boldsymbol{\nabla}\left(p+\frac{1}{2}|\mathbf{u}|^{2}+\dot{\phi}\right) \\
& =\mathbf{u} \times \boldsymbol{\omega}-\nu \boldsymbol{\nabla} \times \boldsymbol{\omega}+\boldsymbol{\nabla}\left(p_{\omega}+\frac{1}{2}\left|\mathbf{u}_{\omega}\right|^{2}+\mathbf{u}_{\omega} \cdot \mathbf{u}_{\phi}\right),
\end{aligned}
$$

where in the second line the Bernoulli equation (3.6) is invoked. This version of the potential-vortical formulation is more physically intuitive in terms of vortex dynamics and it is our main tool in this work. However, this version contains expressions such as $\mathbf{u} \times \boldsymbol{\omega}$ which are hard to give rigorous meaning when $\operatorname{Re} \rightarrow \infty$, and thus the conservation form (3.14) is preferred in considering the infinite-Reynoldsnumber limit.

As should be clear from the review in Sec. II, the detailed Josephson-Anderson relation involves as well the conservation of vorticity and kinetic energy. The equation expressing local conservation of vorticity can be obtained in the potential-vortical formulation by taking the curl of Eq. (3.16). It has the same form as Eq. (2.5) with the vorticity-flux tensor $\boldsymbol{\Sigma}$ as given in Eq. (2.3) with $\mathbf{g} \equiv \mathbf{0}$. This is, of course, just the usual Helmholtz equation.

The equation for the local conservation of the kinetic energy of the rotational flow can be obtained by dotting $\mathbf{u}_{\omega}$ into Eq. (3.16), which yields

$$
\begin{aligned}
& \partial_{t}\left(\frac{1}{2}\left|\mathbf{u}_{\omega}\right|^{2}\right)+\boldsymbol{\nabla} \cdot\left[\left(p_{\omega}+\frac{1}{2}\left|\mathbf{u}_{\omega}\right|^{2}+\mathbf{u}_{\omega} \cdot \mathbf{u}_{\phi}\right) \mathbf{u}_{\omega}-\nu \mathbf{u} \times \boldsymbol{\omega}\right] \\
& \quad=-\mathbf{u}_{\phi} \cdot(\mathbf{u} \times \boldsymbol{\omega}-\nu \boldsymbol{\nabla} \times \boldsymbol{\omega})-\nu|\boldsymbol{\omega}|^{2} .
\end{aligned}
$$

Note that the $\mathbf{u}_{\phi} \cdot(\nu \boldsymbol{\nabla} \times \boldsymbol{\omega})$ term is needed on the righthand side of Eq. (3.17) so that $\nu \mathbf{u} \times \boldsymbol{\omega}$ appears in the square bracket on the left-hand side. Otherwise, the term inside the square bracket would be $\nu \mathbf{u}_{\omega} \times \omega$, whose normal component does not vanish on the surface $\partial B$ of the object. Since the expression in the square bracket is a spatial energy flux, it should imply a vanishing flux through the surface. A corresponding equation can be obtained also for the interaction energy of potential and vortical flow by dotting $\mathbf{u}_{\phi}$ into Eq. (3.16):

$$
\begin{aligned}
& \partial_{t}\left(\mathbf{u}_{\phi} \cdot \mathbf{u}_{\omega}\right)+\boldsymbol{\nabla} \cdot\left[\left(p_{\omega}+\frac{1}{2}\left|\mathbf{u}_{\omega}\right|^{2}+\mathbf{u}_{\omega} \cdot \mathbf{u}_{\phi}\right) \mathbf{u}_{\phi}\right. \\
& \left.\quad+\left(p_{\phi}+\frac{1}{2}\left|\mathbf{u}_{\phi}\right|^{2}\right) \mathbf{u}_{\omega}\right]=+\mathbf{u}_{\phi} \cdot(\mathbf{u} \times \boldsymbol{\omega}-\nu \boldsymbol{\nabla} \times \boldsymbol{\omega}) .
\end{aligned}
$$

The equal and opposite terms on the right-hand sides of Eqs. (3.17) and (3.18) clearly represent energy transfer between rotational and potential flow. Note that the triple product $\mathbf{u}_{\phi} \cdot(\mathbf{u} \times \boldsymbol{\omega})$ can be rewritten as $\mathbf{u}_{\phi} \cdot\left(\mathbf{u}_{\omega} \times \boldsymbol{\omega}\right)$ using $\mathbf{u}=\mathbf{u}_{\omega}+\mathbf{u}_{\phi}$, so that only self-advection of vorticity by the rotational motions themselves contributes to transfer.

Because of the cancellation of these two terms, the sum of the rotational and interaction energies satisfies the equation

$$
\partial_{t}\left(\frac{1}{2}\left|\mathbf{u}_{\omega}\right|^{2}+\mathbf{u}_{\phi} \cdot \mathbf{u}_{\omega}\right)+\boldsymbol{\nabla} \cdot\left[\left(p_{\omega}+\frac{1}{2}\left|\mathbf{u}_{\omega}\right|^{2}+\mathbf{u}_{\omega} \cdot \mathbf{u}_{\phi}\right) \mathbf{u}+\left(p_{\phi}+\frac{1}{2}\left|\mathbf{u}_{\phi}\right|^{2}\right) \mathbf{u}_{\omega}-\nu \mathbf{u} \times \boldsymbol{\omega}\right]=-\nu|\boldsymbol{\omega}|^{2} .
$$

Importantly, this combination of energies is conserved for $\nu \rightarrow 0$, as long as solutions remain smooth in the limit. We refer to the space integral $E_{R}(t)=\rho \int_{\Omega}\left[\frac{1}{2}\left|\mathbf{u}_{\omega}\right|^{2}+\mathbf{u}_{\omega} \cdot \mathbf{u}_{\phi}\right]$ as relative kinetic energy, because it corresponds to the total kinetic energy $E(t)=\rho \int_{\Omega} \frac{1}{2}|\mathbf{u}|^{2}$ of Navier-Stokes solution u measured relative to the (conserved) kinetic energy $E_{\phi}=\rho \int_{\Omega} \frac{1}{2}\left|\mathbf{u}_{\phi}\right|^{2}$ of Euler solution $\mathbf{u}_{\phi}$. Of course, this correspondence is purely formal, since in the body frame $E(t)=+\infty$ and $E_{\phi}=+\infty$ separately. It is worth emphasiz- ing that the relative kinetic energy $E_{R}(t)$ is sign indefinite and may take on negative values.

The detailed Josephson-Anderson relation for confined channel flow that is considered in Sec. II involves the global balances of kinetic energy, not the local ones. Before we can derive the analog of that relation for our problem, we must consider vorticity generation at the body surface and the precise far-field asymptotics of the rotational velocity field. 


\section{Generation of vorticity at the boundary}

In order to make certain that our problem is physically meaningful, we must consider an issue neglected until now, namely, the acceleration of the body from rest to constant velocity. Here it is more natural to consider the body as moving and the fluid as at rest at infinity. This relative motion can be accomplished by a translational acceleration protocol a $(t)$ which over some time interval $0 \leq t \leq T$ takes the body from zero velocity to velocity $\mathbf{V}$, including the possibility of an impulsive acceleration $\mathbf{a}(t)=\mathbf{V} \delta(t)$ with $T=0$. The Lighthill vorticity source for this situation is of the form [77]

$$
\boldsymbol{\sigma}=-\hat{\mathbf{n}} \times[\mathbf{a}(t)+\nabla p],
$$

where it is assumed that $\mathbf{a}(t) \equiv \mathbf{0}$ for $t>T$. It follows that vorticity is created only tangential to the body surface and, after the time $T$, is generated along the surface isobars or pressure isolines [19]. Here it is appropriate to note that, because of the stick boundary conditions on the velocity, $\hat{\mathbf{n}} \cdot \boldsymbol{\omega}=0$ everywhere on the surface of any nonrotating body $B$. Thus, vortex lines on solid surfaces in classical Newtonian fluids lie, in general, parallel to the surface $\partial B$. Vortex lines can terminate only on a solid (nonrotating) wall at some exceptional points where $\boldsymbol{\omega}=\mathbf{0}$, which are possible points of boundary-layer separation [19]. This behavior at walls is an important difference from superfluids, where quantized vortex lines can often terminate at a solid surface and, when they do so, intersect it almost normally so as to satisfy the no-flow-through condition [84]. Vortex half rings can in fact be observed standing at the surface of a body moving through a superfluid (e.g., see Fig. 2 in Ref. [31]). On the contrary, vorticity is generated principally parallel to the surface in the classical case, as closed vortex loops encircling the body.

If the fluid is initially at rest or, more generally, has zero net vorticity, then this condition is preserved in time:

$$
\int_{\Omega} \omega d V=\mathbf{0}
$$

This result is known as Föppl's theorem [85], but the standard proof (e.g., see Ref. [70], p. 74) assumes some sufficient decay of the vorticity at infinity, which is a priori unknown in our problem. It is therefore important and illuminating to give a direct proof based upon Lighthill's theory by showing that

$$
\frac{d}{d t} \int_{\Omega} \boldsymbol{\omega} d V=-\int_{\partial B} \hat{\mathbf{n}} \times[\mathbf{a}(t)+\nabla p] d A=\mathbf{0} .
$$

The first term involving the spatially uniform acceleration $\mathbf{a}(t)$ easily vanishes due to the elementary result [70]

$$
\int_{\partial B} \hat{\mathbf{n}} d A=\mathbf{0}
$$

The term involving the pressure gradient can be rewritten as

$$
\int_{\partial B} \hat{\mathbf{n}} \times \boldsymbol{\nabla} p d A=\int_{\partial B} \hat{\mathbf{t}}|\nabla p| d A
$$

where

$$
\hat{\mathbf{t}}=\hat{\mathbf{n}} \times \frac{\boldsymbol{\nabla} p}{|\boldsymbol{\nabla} p|}=\frac{d \mathbf{x}}{d s}(p, s)
$$

is the unit tangent vector to surface isobars, and $\mathbf{x}(p, s)$ is a smooth parametrization of the isobar with pressure value $p$, in terms of arc length $s$ along the isobar. Here we appeal to the Sard theorem of differential topology which implies for a smooth pressure field $p(\mathbf{x}, t)$ that, for almost every $p$, the connected components of the surface isobar with that $p$ value are simple closed smooth curves on $\partial B$ [86]. Then, using the standard result

$\int_{\partial B} \delta(p(\mathbf{x}, t)-p) d A=\int_{\{\mathbf{x}: p(\mathbf{x}, t)=p\}} \frac{d s}{|\nabla p(\mathbf{x}(p, s), t)|}$

known in mathematics as the coarea formula [87], it follows that

$$
\int_{\partial B} \hat{\mathbf{n}} \times \boldsymbol{\nabla} p d A=\int_{\partial B} \hat{\mathbf{t}}(p, s) d p d s=\int d p \int d s \frac{d \mathbf{x}}{d s}=\mathbf{0}
$$

since the isobars are closed curves for almost every $p$ value.

\section{Far-field velocities and vortex impulse}

With the previous results in hand, we now develop asymptotic formulas for the velocities $\mathbf{u}_{\omega}(\mathbf{x}, t)$ and $\mathbf{u}_{\phi}(\mathbf{x}, t)$ in the far field or for large $r=|\mathbf{x}|$.

We begin with the rotational velocity field $\mathbf{u}_{\omega}$. Here we follow closely an argument of Cantwell for a different problem of forced jets [88] by considering the vector potential that appears in the Helmholtz formula (3.12)

$\boldsymbol{\psi}_{\omega}(\mathbf{x}, t)=\int_{\Omega} \frac{\boldsymbol{\omega}\left(\mathbf{x}^{\prime}, t\right)}{4 \pi\left|\mathbf{x}-\mathbf{x}^{\prime}\right|} d V^{\prime}+\int_{\partial B} \frac{\hat{\mathbf{n}} \times \mathbf{u}_{\omega}\left(\mathbf{x}^{\prime}, t\right)}{4 \pi\left|\mathbf{x}-\mathbf{x}^{\prime}\right|} d A^{\prime}$,

so that $\mathbf{u}_{\omega}=\boldsymbol{\nabla} \times \boldsymbol{\psi}_{\omega}$. As in Ref. [88], we make a multipole expansion using $\left(1 /\left|\mathbf{x}-\mathbf{x}^{\prime}\right|\right)=(1 / r)+\left(\mathbf{x} \cdot \mathbf{x}^{\prime} / r^{3}\right)+\cdots$ obtaining

$$
\boldsymbol{\psi}_{\omega}(\mathbf{x}, t)=\frac{\mathbf{q}_{\omega}(t)}{4 \pi r}+\frac{\mathbf{I}_{\omega}(t) \times \mathbf{x}}{4 \pi r^{3}}+O\left(\frac{1}{r^{3}}\right) \cdots,
$$


where we introduce the total vorticity

$$
\mathbf{q}_{\boldsymbol{\omega}}(t)=\int_{\Omega} \boldsymbol{\omega}(\mathbf{x}, t) d V+\int_{\partial B} \hat{\mathbf{n}} \times \mathbf{u}_{\omega}(\mathbf{x}, t) d A,
$$

including the contribution from the surface vortex sheet and also the corresponding vortex impulse [21,79]

$\mathbf{I}_{\omega}(t)=\frac{1}{2}\left[\int_{\Omega} \mathbf{x} \times \boldsymbol{\omega}(\mathbf{x}, t) d V+\int_{\partial B} \mathbf{x} \times\left[\hat{\mathbf{n}} \times \mathbf{u}_{\omega}(\mathbf{x}, t)\right] d A\right]$.

We first note that $\mathbf{q}_{\omega}(t) \equiv \mathbf{0}$ so that the "monopole" term in Eq. (3.27) is zero. The volume integral in the definition (3.28) of $\mathbf{q}_{\omega}$ vanishes by the results of the previous Sec. III C. Furthermore, by the boundary condition for $\mathbf{u}_{\omega}$ on $\partial B$,

$$
\int_{\partial B} \hat{\mathbf{n}} \times \mathbf{u}_{\omega}(\mathbf{x}, t) d A=-\int_{\partial B} \hat{\mathbf{n}} \times \nabla \phi(\mathbf{x}, t) d A=\mathbf{0}
$$

by precisely the same argument as in Sec. III C.

We thus obtain by a curl of Eq. (3.27) the final result

$$
\mathbf{u}_{\omega}(\mathbf{x}, t) \underset{|\mathbf{x}| \rightarrow \infty}{\sim} \frac{-\mathbf{I}_{\omega}(t) r^{2}+3\left[\mathbf{I}_{\omega}(t) \cdot \mathbf{x}\right] \mathbf{x}}{4 \pi r^{5}},
$$

which is a dipole field. Quite intuitively, the vortical wake behind the body appears at very large distances like a vortex ring with impulse $\mathbf{I}_{\omega}(t)$. The formula (3.31) can be simply rewritten in spherical coordinates for polar angle $\theta$ measured from the positive $x$ axis as

$$
\mathbf{u}_{\omega}(\mathbf{x}, t) \underset{|\mathbf{x}| \rightarrow \infty}{\sim} \frac{\mathrm{I}_{\omega}}{4 \pi r^{3}} \hat{\mathbf{x}}-\frac{3 \mathrm{I}_{\omega} \cos \theta}{4 \pi r^{3}} \hat{\mathbf{r}}
$$

with $\mathbf{I}_{\omega}=-\mathrm{I}_{\omega} \hat{\mathbf{x}}$. The sign here can be simply understood because the vortical wake behind the body must reduce the velocity of the potential flow. Alternatively, in the fluid rest frame, the vortical impulse must be in the direction of motion of the body.

Asymptotics of the potential-flow velocity can be similarly obtained from $\mathbf{u}_{\phi}=\boldsymbol{\nabla} \times \boldsymbol{\psi}_{\phi}$ where

$$
\boldsymbol{\psi}_{\phi}(\mathbf{x}, t)=\int_{\partial B} \frac{\hat{\mathbf{n}} \times \mathbf{u}_{\phi}\left(\mathbf{x}^{\prime}, t\right)}{4 \pi\left|\mathbf{x}-\mathbf{x}^{\prime}\right|} d A^{\prime} .
$$

This velocity to leading order is the constant $V \hat{\mathbf{x}}$ plus a dipole term similar to Eq. (3.31) but involving the vortex impulse $\mathbf{I}_{\phi}(t)$ associated with the surface discontinuity. However, in what follows we need only the leading term, so that

$$
\mathbf{u}_{\phi}(\mathbf{x}, t) \underset{|\mathbf{x}| \rightarrow \infty}{\sim} V \hat{\mathbf{x}}+O\left(\frac{1}{r^{3}}\right)
$$

or equivalently, in terms of the scalar potential

$$
\phi(\mathbf{x}, t) \underset{|\mathbf{x}| \rightarrow \infty}{\sim} \operatorname{Vr} \cos \theta+O\left(\frac{1}{r^{2}}\right) .
$$

\section{E. Global momentum and energy integrals}

Using the asymptotic formulas of the preceding section, we can now study the global integrals of momentum and kinetic energy for our flow.

The total vortex momentum is defined by

$$
\mathbf{P}_{\omega}(t)=\rho \int \mathbf{u}_{\omega}(\mathbf{x}, t) d V
$$

With the dipole asymptotics (3.31) for the integrand, this integral is convergent but only conditionally so. Note that the vortex momentum is not equal to density times vortex impulse. In fact, it is well known that

$$
\mathbf{P}_{\omega}(t)=\frac{2}{3} \rho \mathbf{I}_{\omega}(t)
$$

This result can be shown by an argument of Cantwell [88] using the representation $\mathbf{u}_{\omega}=\boldsymbol{\nabla} \times \boldsymbol{\psi}_{\omega}$ and the identity $\int_{S_{R}}\left(\mathbf{x} / 4 \pi\left|\mathbf{x}-\mathbf{x}^{\prime}\right|\right) d \Omega=\frac{1}{3}\left(\mathbf{x}^{\prime} / R\right)$ for a sphere $S_{R}$ of radius $R>r^{\prime}$. In Appendix B we give another proof.

Total kinetic energy of the vortical wake is clearly well defined by the integral

$$
E_{\omega}(t)=\frac{1}{2} \rho \int_{\Omega}\left|\mathbf{u}_{\omega}(\mathbf{x}, t)\right|^{2} d V,
$$

because the square of the dipole field decays asymptotically as $1 / r^{6}$. However, the total interaction energy

$$
E_{\mathrm{int}}(t)=\rho \int_{\Omega} \mathbf{u}_{\omega}(\mathbf{x}, t) \cdot \mathbf{u}_{\phi}(\mathbf{x}, t) d V
$$

is again at most conditionally convergent and, if convergent, might be expected to vanish. As we recall, the Kelvin minimum-energy theorem is exactly the statement that the potential and vortical velocity fields are orthogonal and, in his discussion of a moving body, Lighthill appealed to this result (see Ref. [19], p. 56). However, he worked in the rest frame of the fluid, where the Kelvin minimum-energy theorem indeed holds, but we work in the rest frame of the body where it does not.

We find instead that

$$
E_{\text {int }}(t)=\mathbf{P}_{\omega}(t) \cdot \mathbf{V}
$$

The proof is simple: Using $\mathbf{u}_{\omega} \cdot \mathbf{u}_{\phi}=\boldsymbol{\nabla} \cdot\left(\phi \mathbf{u}_{\omega}\right)$, we obtain 


$$
\begin{aligned}
E_{\text {int }}(t) & =\lim _{R \rightarrow \infty} \rho \int_{B_{R} \backslash B} \boldsymbol{\nabla} \cdot\left(\phi \mathbf{u}_{\omega}\right) d V \\
& =\lim _{R \rightarrow \infty} \rho \int_{S_{R}} \phi\left(\hat{\mathbf{r}} \cdot \mathbf{u}_{\omega}\right) d A,
\end{aligned}
$$

where $B_{R}$ is the ball of radius $R$ centered at the origin so that $\partial B_{R}=S_{R}$. Note that the contribution from the body surface $\partial B$ vanishes because $\hat{\mathbf{n}} \cdot \mathbf{u}_{\omega}=0$ there. Using the asymptotics Eqs. (3.32) and (3.35) for $\mathbf{u}_{\omega}$ and $\phi$ give the integral over solid angle $d \Omega=\sin \theta d \theta d \varphi$ :

$$
\begin{aligned}
E_{\text {int }}(t) & =\lim _{R \rightarrow \infty} \rho \int_{S_{R}} V R \cos \theta\left(-\frac{I_{\omega} \cos \theta}{2 \pi R^{3}}\right) R^{2} d \Omega \\
& =-\frac{2}{3} \rho I_{\omega} V=\frac{2}{3} \rho \mathbf{I}_{\omega} \cdot \mathbf{V}=\mathbf{P}_{\omega} \cdot \mathbf{V}
\end{aligned}
$$

We conclude finally that the relative kinetic energy in the body frame is given by

$$
E_{R}(t)=E_{\omega}(t)+\mathbf{P}_{\omega}(t) \cdot \mathbf{V},
$$

a result that should be expected by Galilean invariance. This conclusion will appear very familiar to superfluid physicists, since a similar result holds for a vortex ring moving in unbounded space $[4,89]$. However, in that case $E_{\text {int }}=\rho \mathbf{I}_{\omega} \cdot \mathbf{V}$ in our notations [90]; i.e., it is a vortex impulse which appears rather than a vortex momentum.

\section{F. Global balances of momentum and energy}

We can now derive the balance equations for the global integrals in Sec. IIIE by integrating the local balance equations over $\Omega$. Integrating Eq. (3.14) gives the global momentum balance as

$$
\frac{d \mathbf{P}_{\omega}}{d t}=\mathbf{F},
$$

where

$$
\mathbf{F}=\int_{\partial B}\left(P_{\omega} \hat{\mathbf{n}}-2 \eta \mathbf{S} \hat{\mathbf{n}}\right) d A
$$

is the total force applied to the fluid by the body. Note that all contributions from infinity are vanishing because of the asymptotic decay of the momentum flux as $r^{-3}$ from (3.32) and (3.34). This force can be rewritten also as

$$
\mathbf{F}=\int_{\partial B}\left(P_{\boldsymbol{\omega}} \hat{\mathbf{n}}+\eta \hat{\mathbf{n}} \times \boldsymbol{\omega}\right) d A
$$

since the viscous Newtonian stress vector at the wall $\tau_{w}=$ $-2 \eta \mathbf{S} \hat{\mathbf{n}}$ is related to the wall vorticity $\boldsymbol{\omega}_{w}$ by $\boldsymbol{\tau}_{w}=\eta \boldsymbol{\omega}_{w} \times \hat{\mathbf{n}}$ $[19,70]$. An important consequence of Eq. (3.44) is that the total momentum of the vortical wake increases monotonically in time. In particular, there can be no global statistical steady state for this flow.

Integrating Eq. (3.18) gives the global balance of the interaction energy as

$$
\frac{d E_{\text {int }}}{d t}=+\rho \int_{\Omega} \mathbf{u}_{\phi} \cdot(\mathbf{u} \times \boldsymbol{\omega}-\nu \boldsymbol{\nabla} \times \boldsymbol{\omega}) d V,
$$

where the decay of the energy flux asymptotically as $r^{-3}$ implies no contribution from infinity, and the no-flowthrough condition implies no contribution from the body surface. On the other hand, directly differentiating expression (3.42) for interaction energy and using the global momentum balance (3.44) gives [91]

$$
\frac{d E_{\mathrm{int}}}{d t}=\mathbf{F} \cdot \mathbf{V}
$$

Although $\mathbf{F}$ must fluctuate in time, the dot product above will generally be negative, as the force applied by the body opposes the fluid flow. Thus, drag appears as loss of energy of the potential flow due to negative work by the body on the fluid. Note that $E_{\text {int }}(t)$ is monotonically decreasing, but the energy $E_{\text {int }}(t)+E_{\phi}$ does not decrease, of course, because there is an infinite reservoir of kinetic energy in the potential flow. The energy is transferred to the vortical flow, as can be seen by integrating Eq. (3.17) in like fashion over $\Omega$ to give

$$
\frac{d E_{\omega}}{d t}=-\rho \int_{\Omega} \mathbf{u}_{\phi} \cdot(\mathbf{u} \times \boldsymbol{\omega}-\nu \boldsymbol{\nabla} \times \boldsymbol{\omega}) d V-\int_{\Omega} \eta|\omega|^{2} d V .
$$

The energy transferred to the rotational fluid motions is ultimately dissipated by viscosity.

The combination of Eqs. (3.47) and (3.48) yields the most fundamental result of our paper, the classical version of the detailed Josephson-Anderson relation for flow past a solid body written in various equivalent forms as

$$
\begin{aligned}
-\mathbf{F} \cdot \mathbf{V} & =-\rho \int_{\Omega} \mathbf{u}_{\phi} \cdot(\mathbf{u} \times \boldsymbol{\omega}-\nu \boldsymbol{\nabla} \times \boldsymbol{\omega}) d V \\
& =-\int d J \int(\mathbf{u} \times \boldsymbol{\omega}-\nu \boldsymbol{\nabla} \times \boldsymbol{\omega}) \cdot d \ell \\
& =-\frac{1}{2} \int d J \int \epsilon_{i j k} \Sigma_{i j} d \ell_{k},
\end{aligned}
$$

where the second two expressions follow the notations in Sec. II. The relation (3.50) expresses an instantaneous balance between power injected by the drag force $\mathbf{F}$ acting back on the fluid and the vorticity flux crossing the mass current along potential-flow lines. This result should be compared with the detailed Josephson-Anderson relation (2.13) derived by Huggins, which expresses an instantaneous equality between the rate of work done on the ideal 
potential flow by the total pressure $h^{\prime}$ and the vorticity flux across the mass current, which thereby transfers that energy to vortical motions, exactly as here.

Although the momentum of the vortical motions is constantly increasing, Eq. (3.49) makes it plausible that the rotational flow energy should have a long-time steadystate limit, sufficiently long after the initial acceleration of the body when the potential-flow solution $\mathbf{u}_{\phi}$ becomes time independent. In fact, it is generally expected that the entire flow within any fixed distance of the body, after a sufficiently long time that depends upon the distance, shall reach a steady state whose character depends upon the Reynolds number, with a deterministic stationary flow at low Reynolds numbers, then periodic flow, and finally a chaotic flow with a long-time ergodic behavior at sufficiently high Reynolds number. Of course, there may be multiple distinct stable regimes, each attracting some domain of initial conditions. We refer to these hypothesized quasisteady regimes in some vicinity of the body as the local steady states. At any finite time, however, one can observe at some far distance downstream a time-dependent flow with increasing momentum.

Denoting the suitable time average for such a local steady regime as $\langle\cdot\rangle$ and assuming that total vortical energy does in fact achieve a long-time mean value, we can then average Eqs. (3.49) and (3.50) to obtain a steady-state version of the Josephson-Anderson relation as

$$
\begin{aligned}
-\langle\mathbf{F}\rangle \cdot \mathbf{V} & \doteq-\rho \int_{\Omega^{\prime}}\left\langle\mathbf{u}_{\phi} \cdot(\mathbf{u} \times \boldsymbol{\omega}-\nu \boldsymbol{\nabla} \times \boldsymbol{\omega})\right\rangle d V \\
& \doteq \eta \int_{\Omega^{\prime}}\left\langle|\boldsymbol{\omega}|^{2}\right\rangle d V
\end{aligned}
$$

where $\Omega^{\prime} \subset \Omega$ has here been chosen large enough so that the space integrals over $\Omega^{\prime}$ and $\Omega$ agree to any desired precision [92] denoted by "․, " and then time averages are taken over a long enough interval to obtain a steady state within the region $\Omega^{\prime}$. The relation (3.51) expresses equality in the mean of three distinct quantities: the power input by the drag force, the energy transfer from potential to rotational motions by vortex motion, and the viscous energy dissipation. The third expression is clearly non-negative, expressing the time irreversibility of the viscous NavierStokes dynamics. It follows that the drag force must generally oppose the free-stream velocity, just as expected. The middle expression in Eq. (3.51) involves the vector quantity

$$
\mathbf{f}_{v}=\rho(\mathbf{u} \times \boldsymbol{\omega}-\nu \boldsymbol{\nabla} \times \boldsymbol{\omega}),
$$

which is sometimes called the vortex force, and, intuitively, drag is associated with the vortex force opposing the potential flow. An even more useful expression is

$$
-\langle\mathbf{F}\rangle \cdot \mathbf{V}=-\frac{1}{2} \int d J \int \epsilon_{i j k}\left\langle\Sigma_{i j}\right\rangle d \ell_{k}
$$

which represents mean drag in terms of vorticity flux crossing the potential mass current. In a local steady state, mean vorticity flux further satisfies the relation dual to Eq. (2.6)

$$
\left\langle\Sigma_{i j}\right\rangle=\epsilon_{i j k} \partial_{k}\langle h\rangle,
$$

implying also that $\partial_{j}\left\langle\Sigma_{i j}\right\rangle=0$. Together, the two relations (3.53) and (3.54) very strongly constrain the vortex dynamics and statistics that contribute to the mean drag.

The detailed relation (3.50) holds, of course, even before a local steady regime is achieved (but after the period of initial acceleration). One simple general deduction can be made from this principle by substituting the equation of motion (3.16) into the energy-transfer term to obtain [93]

$$
\begin{aligned}
-\mathbf{F} \cdot \mathbf{V} & =-\rho \int_{\Omega} \mathbf{u}_{\phi} \cdot(\mathbf{u} \times \boldsymbol{\omega}-\nu \boldsymbol{\nabla} \times \boldsymbol{\omega}) d V \\
& =-\rho \int_{\Omega} \mathbf{u}_{\phi} \cdot\left(\dot{\mathbf{u}}_{\omega}+\boldsymbol{\nabla} h^{\prime}\right) d V
\end{aligned}
$$

with $h^{\prime}=p+\frac{1}{2}|\mathbf{u}|^{2}+\dot{\phi}$ as in Eq. (2.13). By applying the same arguments as those used to evaluate $\int_{\Omega} \mathbf{u}_{\phi} \cdot \mathbf{u}_{\omega} d V$ in Sec. III E,

$$
-\rho \int_{\Omega} \mathbf{u}_{\phi} \cdot \dot{\mathbf{u}}_{\omega} d V=-\dot{\mathbf{P}}_{\omega}(t) \cdot \mathbf{V}=-\mathbf{F} \cdot \mathbf{V}
$$

the consequence is that

$$
\rho \int_{\Omega} \mathbf{u}_{\phi} \cdot \boldsymbol{\nabla} h^{\prime} d V=\int d J\left(\Delta h^{\prime}\right)=0
$$

with the quantity

$\Delta h^{\prime}=\int \nabla h^{\prime} \cdot d \ell=h^{\prime}(\ell=+\infty)-h^{\prime}(\ell=-\infty)$

given as an integral along each streamline and representing the drop in the total pressure along this entire line. It seems certain that $\Delta h^{\prime} \leq 0$, since the presence of the body should cause a pressure drop and a reduced streamwise velocity in its wake. The conclusion from Eq. (3.57) is that $\Delta h^{\prime}=0$ along all of these lines, so that the total pressure recovers completely from whatever drop it experienced by the presence of the body. This result underlines the great difference from channel flow, where the detailed Josephson-Anderson relation (2.13) of Huggins involves only the total pressure head in the channel. 


\section{FLOW PAST A SPHERE}

To make the preceding discussion more concrete, we consider in this section the special case of flow past a sphere. The rich phenomenology of this flow has been the subject of a recent review [94], which classifies the flow into eight regimes as a function of increasing Reynolds number: (i) the axisymmetric wake regime, (ii) the planar symmetric wake regime with a counterrotating pair of trailing streamwise vortices, (iii) the shedding regime with alternating hairpin vortices, (iv) a regime with separating vortex tubes due to Kelvin-Helmholtz instability of the separated boundary layer, (v) the subcritical regime where the point of instability moves upstream closer to the sphere, (vi) the critical regime of "drag crisis" with reattachment of the boundary layer and formation of a laminar separation bubble, (vii) the supercritical regime in which the bubble shrinks or disappears, and finally (viii) the transcritical regime, which is an apparently asymptotic state with constant mean drag. The detailed Josephson-Anderson relation (3.50) holds in all of these regimes and reveals that, despite the very substantial differences in flow physics between the different regimes, the mechanism of drag in terms of vortex dynamics is intrinsically the same for all of them.

\section{A. Josephson-Anderson relation for a sphere}

We give here the general relation (3.50) a concrete form for flow around a sphere of radius $a$. The first important ingredient of that relation is the inviscid flow solution $\mathbf{u}_{\phi}$. This is well known ([69], Sec. VI. 8) to be given in spherical coordinates $(r, \theta, \varphi)$ by the scalar potential

$$
\phi=V\left(r+\frac{a^{3}}{2 r^{2}}\right) \cos \theta,
$$

where, once again, the zenith for measurement of the polar angle $\theta$ is the positive streamwise direction. This formula implies the potential-flow velocity

$u_{\phi r}=V\left(1-\frac{a^{3}}{r^{3}}\right) \cos \theta, \quad u_{\phi \theta}=-V\left(1+\frac{a^{3}}{2 r^{3}}\right) \sin \theta$,

whose streamlines are plotted in Fig. 4 for an axial plane at fixed azimuthal angle $\varphi=0$. It is even more useful to represent this flow by the Stokes stream function ([69], Sec. II. 2) given both in spherical coordinates and in cylindrical coordinates $(\sigma, \varphi, x)$ as

$$
\begin{aligned}
\psi & =\frac{1}{2} V r^{2} \sin ^{2} \theta\left(1-\frac{a^{3}}{r^{3}}\right) \\
& =\frac{1}{2} V \sigma^{2}\left[1-\frac{a^{3}}{\left(\sigma^{2}+x^{2}\right)^{3 / 2}}\right]
\end{aligned}
$$

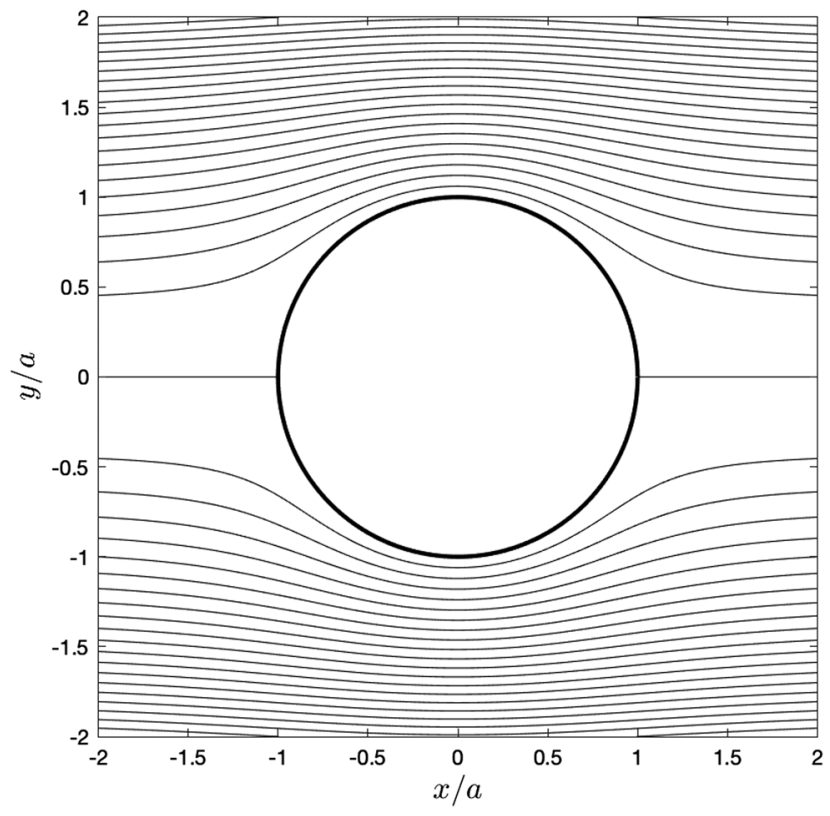

FIG. 4. Streamlines of the ideal flow around a sphere.

([69], Sec. VI. 8) so that

$$
u_{\phi x}=\frac{1}{\sigma} \frac{\partial \psi}{\partial \sigma}, \quad u_{\phi \sigma}=-\frac{1}{\sigma} \frac{\partial \psi}{\partial x} .
$$

Here we follow the fluid mechanics literature in denoting $\sigma=\sqrt{y^{2}+z^{2}}$ to avoid confusion with mass density $\rho$. Note that streamlines are uniquely identified by the values of stream function $\psi$ and azimuthal angle $\varphi$.

Although not needed for the Josephson-Anderson relation, it is worth recalling that the pressure $p_{\phi}$ for the ideal Euler solution $\mathbf{u}_{\phi}$ follows from the Bernoulli equation (3.6) as

$p_{\phi}=p_{\infty}+\frac{1}{4} V^{2}\left(1-\frac{5 a^{3}}{4 r^{3}}\right) \frac{a^{3}}{r^{3}}+\frac{3}{4} V^{2}\left(1-\frac{a^{3}}{4 r^{3}}\right) \frac{a^{3}}{r^{3}} \cos (2 \theta)$,

and, in particular, on the surface of the sphere:

$$
p_{\phi}(a, \theta)=p_{\infty}-\frac{1}{6} V^{2}+\frac{9}{16} V^{2} \cos (2 \theta) .
$$

This ideal pressure distribution is perfectly symmetrical, with maximum value equal to $p_{\infty}+\frac{1}{2} V^{2}$, the stagnation pressure, at $\theta=0, \pi$ and minimum $p_{\infty}-\frac{1}{16} V^{2}$ at $\theta=\pi / 2$. This symmetry of $p_{\phi}$ around $\theta=\pi / 2$ explains, of course, the vanishing drag for ideal flow past a sphere.

We can now use the above results to develop a more concrete expression for the Josephson-Anderson relation (3.50) in the flow around a sphere. First, it is useful to recall that the Stokes stream function is defined for any 
axisymmetric flow $\mathbf{u}$ so that $d \psi d \varphi=\mathbf{u} \cdot d \mathbf{A}$ along the streamline labeled by $\psi, \varphi$, (see Ref. [69], Sec. II. 2). Thus, the element of mass flux appearing in Eq. (3.50) is

$$
d J=\rho \mathbf{u}_{\phi} \cdot d \mathbf{A}=\rho d \psi d \varphi .
$$

It is also straightforward to obtain from Eq. (4.3) an explicit parametrization of the streamlines with fixed $\psi$ in the form $x= \pm x(\sigma ; \psi)$ for $\sigma_{\min }(\psi)<\sigma<\sigma_{\max }(\psi)$ and with azimuthal angle $\varphi$ constant independent of $\sigma$. However, for the qualitative arguments that we make in the next section, the plots of the streamlines in Fig. 4 are more useful than these analytical expressions.

We shall furthermore require in the next section a projected version of the vorticity conservation equation (2.5) for the azimuthal vorticity $\omega_{\varphi}$, which we see is the most crucial component of vorticity for origin of drag. Here we follow a general idea of Huggins [16], who observed that one can obtain a balance in any plane for the out-of-plane vorticity $\omega_{n}$ by dotting Eq. (2.5) on the right with the unit vector $\hat{\mathbf{N}}$ normal to the plane. The application of this idea to $\omega_{\varphi}$ involves one subtlety, however: The sets of constant $\varphi$ are half planes terminating on the $x$ axis, not full planes. Thus, dotting Eq. (2.5) with the unit vector $\hat{\mathbf{N}}=\widehat{\boldsymbol{\varphi}}$ for one such half plane at constant $\varphi$ yields a planar conservation law

$$
\partial_{t} \omega_{n}+\nabla \cdot \mathbf{j}_{n}=0
$$

in the entire plane normal to $\hat{\mathbf{N}}$, with

$$
\omega_{n}=\boldsymbol{\omega} \cdot \hat{\mathbf{N}}, \quad \mathbf{j}_{n}=\boldsymbol{\Sigma} \cdot \hat{\mathbf{N}} .
$$

Note that $\mathrm{j}_{n k}=\epsilon_{k n l}(\mathbf{u} \times \boldsymbol{\omega}-\nu \boldsymbol{\nabla} \times \boldsymbol{\omega})_{l}$ lies in the plane. However, only the upper part of this plane corresponds to azimuthal angle $\varphi$, whereas the lower part corresponds instead to the half plane with azimuthal angle $\varphi+\pi$ and whose normal vector is $\widehat{\boldsymbol{\varphi}}=-\hat{\mathbf{N}}$. Thus, only in the upper half plane does $\omega_{n}=\omega_{\varphi}$, whereas in the lower half plane $\omega_{n}=-\omega_{\varphi}$. We see that the conservation laws (4.8), which hold separately for each plane through the $x$ axis, are very useful for elucidating the physics.

\section{B. Physical consequences}

We now exploit the Josephson-Anderson relation (3.50) and its time-averaged form Eq. (3.53) in order to develop an exact but qualitative picture of the origin of drag in terms of vortex dynamics. Although our picture has the nature of a "cartoon" which ignores many complex details of the flow in its different regimes (i)-(viii), we argue that it describes the essence of the phenomenon. The concrete predictions that we make should be verifiable empirically in each regime, realized somewhat differently by the specific flow

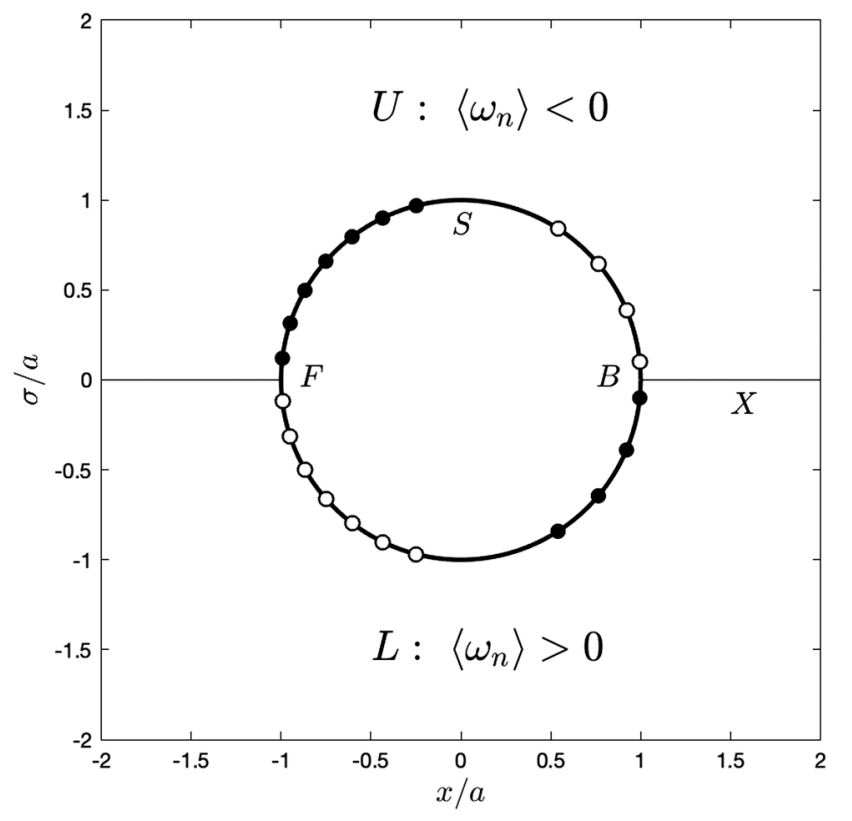

FIG. 5. Schematic of azimuthal vorticity generation on the surface of the sphere. Following the convention of Huggins [16], we use white circles to denote vorticity out of the plane $\left(\omega_{n}>0\right)$ and black circles to denote vorticity into the plane $\left(\omega_{n}<0\right)$. The mean normal (azimuthal) vorticity in the upper half plane $U$ is negative and the mean normal (antiazimuthal) vorticity in the lower half plane $L$ is positive, implying a pole-to-pole asymmetry in the pressure distribution on the surface $S$ of the sphere. The pressure drop along the surface of the sphere from $F$ to $B$ is exactly compensated by the pressure rise from $B$ to infinity along the direction of the positive streamwise axis $X$.

features that are characteristic of that regime which determine the drag quantitatively.

An important general deduction from Eq. (3.50) is that vorticity $\boldsymbol{\omega} \| \mathbf{u}_{\phi}$ does not contribute directly to drag, and, furthermore, that vorticity flux in the directions of $\mathbf{u}_{\phi}$ or $\boldsymbol{\omega}$ do not contribute. As can be seen from Fig. $4, \mathbf{u}_{\phi} \propto \hat{\mathbf{x}}$ to a good approximation already at distances about one radius away from the sphere. Thus, we see that, throughout most of the flow, streamwise vorticity $\omega_{x}$ makes no direct contribution to the drag. Although streamwise vorticity appears in the wake, e.g., in the pair of trailing vortices in regime (ii), these features do not contribute anything to Eq. (3.50). On the other hand, at the surface of the sphere where all flow vorticity is generated and in its close vicinity, $\mathbf{u}_{\phi} \propto \hat{\boldsymbol{\theta}}$ so that it is the polar vorticity $\omega_{\theta}$ which does not contribute to drag in that region. Since all of the vorticity on the sphere is parallel to its surface, it follows that the azimuthal vorticity $\omega_{\varphi}$ plays the crucial role and, in particular, its viscous flux $\Sigma_{r \varphi}$ radially outward. This assertion is our first essential conclusion about the origin of drag in the flow past a sphere.

The next implication of Eq. (3.50) is that outward flux of negative azimuthal vorticity $\omega_{\varphi}<0$ increases drag, whereas flux of positive azimuthal vorticity $\omega_{\varphi}>0$ in fact 
decreases drag. Since the mean power consumption by drag can only be positive [see Eq. (3.51)], it follows that outward flux of negative azimuthal vorticity must be larger in magnitude. In other words, there must be an asymmetry in sign of the azimuthal vorticity on the sphere, with more area and/or stronger magnitudes where $\omega_{\varphi}<0$ and less areas or magnitudes for $\omega_{\varphi}>0$. This situation is illustrated in Fig. 5. According to Lighthill's theory [19], the rate of generation of $\omega_{\varphi}$ is exactly $\sigma_{\varphi}=(1 / a)(\partial p / \partial \theta)$, so that negative azimuthal vorticity is generated by negative or "favorable" pressure gradient, and positive vorticity by positive or "adverse" pressure gradient. We thus conclude that there must be greater area or greater magnitudes of favorable pressure gradient near the front of the sphere than the area or magnitudes of adverse pressure gradient toward the back. This results in a pressure asymmetry unlike that for ideal flow, with the base pressure $p_{B}$ behind the sphere not fully recovering from its drop in the front, thus remaining lower than the stagnation pressure $p_{F}=p_{\infty}+$ $\frac{1}{2} V^{2}$ in the front. Along the sphere surface $S$,

$p_{B}-p_{F}=p(a, 0)-p(a, \pi)=\int_{\pi}^{0} \frac{\partial p}{\partial \theta} d \theta<0$.

Of course, these conclusions are all in agreement with common observations, but it is now seen how they are required for the generation of drag by vortex dynamics.

The picture in Fig. 5 for an axial plane corresponds in three dimensions to generation of azimuthal vortex loops or rings on the surface of the sphere. Those in the front of the sphere have $\omega_{\varphi}<0$, while those toward the rear have mostly $\omega_{\varphi}>0$. As these rings flow radially outward from the surface, they encircle greater amounts of the potential mass flux $J$, with the negative- $\omega_{\varphi}$ rings removing proportionate energy from the potential flow and positive- $\omega_{\varphi}$ rings returning that energy. Since $\omega_{\varphi}<0$ predominates, the net effect is a transfer of kinetic energy from potential to vortical motions, where it is ultimately dissipated by viscosity. In the axisymmetric wake regime (i), this picture is exact, because all vorticity is azimuthal. In higher-Re regimes there will also be some polar vorticity $\omega_{\theta}$ generated on the sphere by azimuthal pressure gradients as $\sigma_{\theta}=-(1 / a)(\partial p / \partial \varphi)$, but these components and their radial flux from the sphere do not contribute to the drag. See Fig. 6 for a rough illustration of the mechanism. It should be noted that this entire picture developed on the basis of the relation (3.50) is consistent with the direct formula (3.46) for the drag force $\mathbf{F}$, since the asymmetry in pressure $P$ and the negative azimuthal vorticity $\omega$ on the surface of the sphere result in pressure and viscous forces both opposing the fluid velocity $\mathbf{V}$.

As the vortex loops grow, they enter the region where $\mathbf{u}_{\phi} \propto \hat{\mathbf{x}}$ and then azimuthal vorticity $\omega_{\varphi}$ is no longer the only relevant component. Indeed, referring now to the

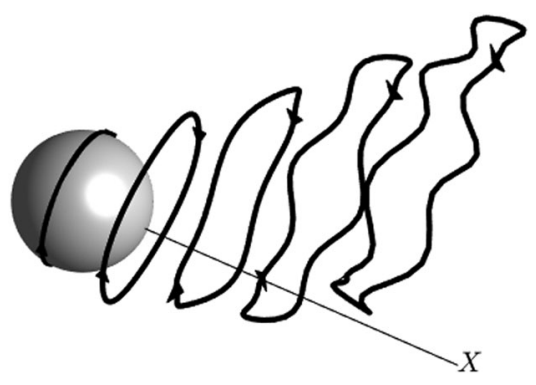

FIG. 6. Rough illustration of the drag mechanism by generation and outward flux of vortex loops with negative azimuthal vorticity. The rings are generated by pressure drop along the surface. As the rings expand outward, they enclose greater mass flux $J$ and subtract proportionate energy from the potential flow. Although the rings initially loop around the streamwise axis $X$, they drift across in time. This implies a flux of azimuthal vorticity across $X$ and allows the pressure to recover far downstream from its drop around the sphere.

cylindrical coordinate system, axial vorticity $\omega_{\sigma}$ appears in this region through the tilting of azimuthal vorticity by the shear in the wake, e.g., as alternating hairpin vortices observed in the shedding regime (iii). It is easy to check that the flux $\Sigma_{\varphi \sigma}$ due to opposite azimuthal motion of the two legs of growing hairpin vortices, one with $\omega_{\sigma}>0$ and the other with $\omega_{\sigma}<0$, contributes also to drag through the relation (3.50) in this spatial region, in addition to the flux $\Sigma_{\sigma \varphi}$ of negative azimuthal vorticity axially outward. In fact, these two effects are basically the same because of the antisymmetry $\Sigma_{\varphi \sigma}=-\Sigma_{\sigma \varphi}$ and both correspond to growth of the tilted vortex rings, which encompass an increasing amount of mass flux $J$ and gain a proportionate amount of energy from the potential flow. This process is the essential mechanism of drag in terms of vortex dynamics for the flow around a sphere as already illustrated in Fig. 6.

Conservation of azimuthal vorticity has one very important additional implication for this process. We show at the end of Sec. III F that $\Delta h^{\prime}=0$ along each streamline of the potential flow. Since now $\dot{\phi}=0, h^{\prime}=h$ and $h(\ell=$ $\pm \infty)=h_{\infty}=p_{\infty}+\frac{1}{2} V^{2}$ at each limit of the streamline. Notice that this value is the same as the stagnation pressure at the front of the sphere $h_{F}=p_{F}=p_{\infty}+\frac{1}{2} V^{2}$ and also that $h_{B}=p_{B}$ at the base point behind the sphere, since the fluid velocity vanishes everywhere on the surface. We thus see that the decrease of $h$ on the surface $S$ of the sphere

$$
\int_{\pi}^{0} \frac{\partial h}{\partial \theta}(a, \theta) d \theta=h_{B}-h_{F}<0
$$

is exactly equal and opposite to the increase of $h$ along the streamwise axis $X$ from the base of the sphere to infinity:

$$
\int_{a}^{\infty} \frac{\partial h}{\partial x}(0, x) d x=h_{\infty}-h_{B}>0 .
$$


See Fig. 5. Since $\left\langle\Sigma_{r \varphi}\right\rangle=(1 / a)(\partial\langle h\rangle / \partial \theta)$ on $S$ and $\left\langle\Sigma_{\sigma \varphi}\right\rangle=(\partial\langle h\rangle / \partial x)$ on $X$, it follows that, on average, the net negative azimuthal vorticity generated on the surface of the sphere is exactly canceled by flux of opposite-signed vorticity across the $x$ axis. This fact was previously noted by Brown and Roshko [95] for flow around a cylinder and by Terrington et al. [96] for flow around a sphere. This exact balance is what permits a steady state to exist with a mean negative value $\left\langle\omega_{\varphi}\right\rangle<0$ in the axial half planes for each fixed value of $\varphi$. The precise origin of the flux across the $x$ axis is still debated, but it is most likely due to lateral motion of shed vortex rings, which initially loop around the $x$ axis but drift across the $x$ axis to become unlinked from it as they advect downstream. See Fig. 6. Although this is a "cartoon picture" assuming simple closed vortex rings, it presumably has an exact counterpart in the actual vortex motions, because solenoidality requires a pairing of the points with $\omega_{\varphi}<0$ on opposite sides of the $x$ axis and both viscous and turbulent diffusion will tend to mix those points from one side to the other.

In this section, we attempt to give a clear and intuitive description of the origin of drag in terms of a schematic picture of the vortex motions. Although qualitative, this picture makes specific predictions in terms of the signs of the vorticity components $\omega_{j}$ and their fluxes $\Sigma_{i j}$, which can be checked empirically by measuring these quantities in numerical simulation or experiment and evaluating their contribution to the drag through the relation (3.50). We note that the numerical study [96] has already used Huggins's vorticity flux (2.3) to illuminate other aspects of the vortex dynamics in the wake behind a sphere, such as tilting of the azimuthal vorticity in the streamwise direction [97].

\section{Superfluid comparisons}

The illustration that we present in the previous section in order to interpret the exact Josphson-Anderson relation (3.50) should be more literally correct for a superfluid, where vorticity is quantized and vortex lines are discrete objects whose motion is objectively defined. In fact, the most fundamental differences between our theoretical results and those for superfluids arise not from the differences between classical and quantum fluids, but instead from the differences between incompressible and compressible. In superfluids, there are generally substantial compressibility effects due to emission of phonons that propagate at the finite speed of sound. Of course, the Josephson-Anderson relation arose in the study of superfluids, and its application there to understanding drag and critical velocities is well established. It is therefore worth reviewing briefly the existing literature in order to point out both the similarities and the differences from classical incompressible fluids.

The pioneer work concerning drag acting on bodies moving through a superfluid is that of Frisch et al. [27], who adopted the zero-temperature Gross-Pitaevskii model to study numerically the motion of a disk at velocity $V$ through a 2D superfluid. We remark that it is straightforward to extend our own analysis for incompressible classical fluids to 2D. Already showing the important effects of compressibility, the authors of Ref. [27] identified the critical velocity $V_{c}$ for appearance of drag to be that for which the local velocity $u$ on the surface of the disk exceeds the sound speed $c_{s}$. At this velocity $V_{c} \doteq 0.44 c_{s}$, quantized vortices are nucleated and emitted as a wake behind the disk. Although compressibility plays a crucial role in their generation, the vortices themselves are incompressible excitations in the superfluid. The picture proposed in Ref. [27] has been further developed in many subsequent works on this same problem [28-30,32-36]. In particular, we note that the generation of the vortices has been verified to occur by the $2 \pi$ phase-slip mechanism [28] and their shedding occurs with the Josephson frequency corresponding to the difference in the generalized chemical potential $\mu_{T}=g n+\frac{1}{2} m u^{2}$ that develops between the exterior flow and the wake behind the disk with low density $n$ and low speed $u$ [29]. Thus, at least for $V<c_{s}$, there is great similarity to the theory that we develop for classical incompressible fluids. Of course, for supersonic motions with $V>c_{s}$, new compressible effects can be observed in superfluids, such as drag by phonon radiation and standing bow waves in front of the disk [30,32], which have no parallel in the incompressible theory developed here.

Studies of superfluid drag have since been extended to 3D, with the superfluid modeled again by Gross-Pitaevskii and the moving object by a suitable time-dependent potential [31,37]. The object was taken in Ref. [31] to be spherical and subject to constant force $\mathbf{F}$, whereas Ref. [37] considered more general ellipsoidal bodies and moving at constant velocity $\mathbf{V}$. The picture emerging from these 3D simulation studies is even more strikingly similar to the one that we derive for classical fluids. In both studies, quantized vortex rings are excited at the surface of the object when it reaches the critical speed $V_{c}$, with the vorticity oriented in the negative azimuthal direction (according to our coordinate conventions). In the case of the body moving at constant speed studied in Ref. [37], the ring vortices are shed into the wake where they grow, extracting energy from the potential flow, and also drift cross-stream so that the asymmetry in the vortex polarity is relaxed far downstream. The observed simulation results are very close to our sketch in Fig. 6. The case with constant applied force $\mathbf{F}$ studied in Ref. [31] shows a bit more complex behavior, because the body decelerates when the vortex ring is emitted. At lower forcing, the quantized vortex ring reattaches to the spherical body and remains pinned there as an arch, legs perpendicular to the surface, even as it continues to grow and expand outward. This regime has no strict classical analog, although it distantly resembles the reattachment of the separating laminar boundary layer observed in the drag crisis of the classical 
regime (vi). At higher forcing, however, the vortex rings completely detach and are shed in the wake, again very similar to our Fig. 6.

Somewhat ironically, the detailed Josephson-Anderson relation was derived by Huggins [10] assuming flow incompressibility, and we are aware of no full extension to the Gross-Pitaevskii model of a superfluid. The original Josephson-Anderson phase relation (1.1) is, of course, directly embodied in the Gross-Pitaevskii equation (with an additional "quantum pressure" term), but this implies no direct connection of vortex motion with energy dissipation. Thus, based on our results in the present paper, we currently have a better understanding of how energy dissipation is associated with vorticity flux for a classical incompressible fluid than we do for quantum superfluids, where the Josephson-Anderson relation originated.

\section{WHY IS IT IMPORTANT?}

To briefly summarize our results in this paper, we derive the detailed Josephson-Anderson relation (3.50) for incompressible fluid flow around a finite solid body, relating drag and energy dissipation to vorticity flux and implying a time-averaged version of Eq. (3.51) valid for the local steady states of the fluid wake. We furthermore discuss in detail the origins of drag in terms of vortex motion for the concrete example of flow past a sphere, obtaining numerous predictions that can be checked empirically. But why are these results important?

We believe first of all that the Josephson-Anderson relation is important because of the theoretical unity that it brings to our understanding of drag for both quantum and classical fluid systems, across all Reynolds-number ranges of the latter case. The relation clearly identifies what is essential for drag in the vortex dynamics and statistics, bypassing all details of secondary importance. In the classical case, these "details" include fascinating phenomena such as boundary-layer separation, formation of streamwise vortices by tilting, transition to turbulence first in the wake and then in the boundary layer, etc. All such details are, of course, crucial in order to determine the drag quantitatively, but only insofar as they modify the primary process: flux of vorticity across the potential mass flux. The precise vortex dynamics and statistics which contribute to the Josephson-Anderson relation must be explained in any quantitative theory of drag.

In addition to the conceptual unification provided by the theory, it also opens up several important new directions of research, which we discuss now in turn.

\section{A. D'Alembert paradox and Onsager anomaly}

The relation (3.50) in particular sheds new light on the d'Alembert paradox $[24,26]$ and opens up the possibility of a novel analysis that relates it directly with Onsager's theory. One crucial observation is that the new potential- vortical formulation (3.14) of incompressible NavierStokes is a local conservation equation for $\rho \mathbf{u}_{\omega}$, the density of "vortex momentum," and it thus has a weak interpretation. We may therefore apply in this formulation the recent techniques to derive Onsager's theory for wallbounded flows [98-102], obtaining necessary conditions for nonvanishing drag and dissipation in the infiniteReynolds-number limit. The relevant limiting Euler solutions must obviously describe rotational flow very distinct from the smooth potential-flow solution. The local energy balances (3.17) and (3.18) make sense also in a weak interpretation and, in the infinite-Re limit, the viscous dissipation appearing in Eq. (3.17) will yield a "dissipative anomaly" like that appearing in the work of Duchon and Robert for periodic domains [103].

The energy-transfer term in Eqs. (3.17) and (3.18) may, however, have a priori no limit because the pointwise product $\mathbf{u}_{\omega} \times \boldsymbol{\omega}$ has no clear meaning when $\mathbf{u}_{\omega}$ and $\boldsymbol{\omega}$ for the limiting Euler solution are merely distributions. Fortunately, this term can be rewritten as a space gradient using the familiar vector calculus identity

$$
\mathbf{u}_{\omega} \times \boldsymbol{\omega}=-\boldsymbol{\nabla} \cdot\left[\mathbf{u}_{\omega} \mathbf{u}_{\omega}-\frac{1}{2}\left|\mathbf{u}_{\omega}\right|^{2} \mathbf{I}\right],
$$

and this is a well-defined distribution even if $\mathbf{u}_{\omega}$ is only square integrable. Because the potential-flow solution $\mathbf{u}_{\phi}$ is smooth, this observation allows us to rewrite the transfer term $\mathcal{T}$ in the Josephson-Anderson relation (3.50) as

$$
\begin{aligned}
\mathcal{T} & =-\rho \int_{\Omega} \mathbf{u}_{\phi} \cdot(\mathbf{u} \times \boldsymbol{\omega}-\nu \boldsymbol{\nabla} \times \boldsymbol{\omega}) d V \\
& =-\rho \int_{\Omega} \boldsymbol{\nabla} \mathbf{u}_{\phi}: \mathbf{u}_{\omega} \mathbf{u}_{\omega} d V+\int_{\partial \Omega} \mathbf{u}_{\phi} \cdot \boldsymbol{\tau}_{w} d A,
\end{aligned}
$$

and, in this form, the relation can be valid even for the limiting weak solutions of Euler equations. See Ref. [104], where it is shown also that the surface contribution from the viscous wall stress $\tau_{w}$ becomes negligible under specific assumptions in the high-Re limit. It is very natural that drag for the limiting Euler solution should be connected with vorticity flux since, as emphasized by Morton [77], generation of vorticity at the wall by tangential pressure gradients is a purely inviscid process.

The connection between the Josephson-Anderson relation (3.50) and the Onsager anomaly for incompressible fluid turbulence [40-42] can, in turn, illuminate the different wall conditions that are experimentally required for observing anomalous energy dissipation in open exterior flows versus those in closed interior flows [48]. In particular, the requirement for wall roughness in the latter class of flows (pipe flows, Taylor-Couette, etc.) needs to be explained. The original Onsager theorem does not shed any light on this puzzle, but simply implies that anomalous dissipation (when it occurs) requires a Hölder singularity of 
velocity with exponent $\leq 1 / 3$. An important clue, however, arises from the empirical observation in simulations [105] and experiments $[66,67]$ that the flow around individual roughness elements exhibits the same characteristic features seen in flow around bluff bodies such as spheres or cylinders, in particular, form drag and separating boundary layers. The term "form drag" in fluid mechanics describes the contribution to the drag force, or force component in the direction of the flow $\mathbf{V}=V \hat{\mathbf{x}}$, which arises in Eq. (3.46) due to the asymmetric pressure distribution $P$ [106] on the body

$$
F_{x}^{\mathrm{form}}=\int_{\partial B} P \hat{n}_{x} d A
$$

In the limit of high Reynolds number, the drag from viscous wall stress slowly vanishes, and the form drag on the roughness elements becomes dominant [105,107]. Of course, it is well known that the drag in wake flows past bluff bodies arises also in the high-Re limit mainly from form drag $[19,46,95]$. On the contrary, the flows that we describe as "closed, interior" could be characterized more accurately as those whose wall geometry does not permit form drag due to the fact that pressure forces act only perpendicular to the wall. In support of this view, we note that developing boundary layers over flat plates are only half-confined, but they permit as well no form drag when the wall is smooth and they are well known to exhibit the same dichotomy as do "internal flows" between vanishing dissipation for smooth walls and anomalous dissipation for rough walls [108].

To reiterate, the only laboratory turbulent flows which exhibit a clear signature of a dissipative anomaly are those with nonvanishing form drag [Eq. (5.3)] and for which the dissipative anomaly is described by the JosephsonAnderson relation (3.50) with vorticity flux from the boundary due to tangential pressure gradients. We know of no counterexample to this general rule [109]. It is therefore consistent with available experimental data to hypothesize that solid walls with form drag and vorticity flux are required to obtain anomalous energy dissipation for 3D incompressible fluids. A possible explanation is that the Hölder singularities required for anomalous dissipation by Onsager's theorem occur for incompressible fluids only in the presence of solid walls. For example, it might be that finite-time incompressible Euler singularities occur only in the presence of walls. However, the best evidence for such Euler singularities is found in simulations in a circular cylindrical domain with smooth walls $[110,111]$, so that this idea does not explain the observed difference between hydraulically smooth and rough walls. A more plausible explanation is that the required singularities do not arise from inviscid Euler dynamics at all, but instead originate by viscous dynamics from thin vortex sheets that are shed into the flow by separating boundary layers which become singular only in the limit $\operatorname{Re} \rightarrow \infty$. In fact, such separating boundary layers are well known to occur in wake flows behind solid bodies $[19,95]$ and are also observed in the wakes behind small roughness elements in a turbulent boundary layer, both by simulation [105] and by experiment $[66,67]$.

The obvious objection to our argument is the strong evidence for anomalous energy dissipation arising from numerical simulations of forced three-dimensional incompressible fluid turbulence in periodic domains [112,113], where there are no walls at all. However, it is a standard practice in such numerical studies to initialize the simulation at high $\mathrm{Re}$ in a Re-dependent manner, as $u_{\operatorname{Re}}(, 0)=$ $u_{\mathrm{Re}^{\prime}}\left(, T^{\prime}\right)$ by using the final state at time $T^{\prime}$ of a smaller Reynolds-number $\operatorname{Re}^{\prime}<\operatorname{Re}$ simulation performed at lower resolution interpolated onto the finer grid of the Re simulation. See Ref. [113], p. L21. This practice of "nested" initialization means that initial conditions $u_{\mathrm{Re}}(, 0)$ have Kolmogorov-type spectra over increasing ranges of scales as Re increases and thus do not correspond to uniformly smooth initial data. Essentially, the numerical simulations are begun from initial conditions that have built-in quasisingularities of the type that would need to be injected by the walls in real-world incompressible turbulent flows. Therefore, current numerical simulations [112,113] do not resolve the issue. To provide definitive evidence one way or the other, numerical simulations must be carried out with smooth initial data fixed independent of Re and then evolved over a fixed time interval at a sequence of Reynolds numbers.

\section{B. New avenues of empirical investigation}

Of more immediate importance for practical applications is the new insight that the Josephson-Anderson relation (3.50) provides on techniques for drag reduction. As discussed previously [16,22], a drag problem occurs not only in turbulent flow at high Reynolds number but also in high-temperature superconductors because, above a critical electric current, quantized magnetic flux lines are nucleated which migrate cross-current and create a voltage drop and effective resistance $[9,13]$. The technological solution that has been found is to introduce some sort of bulk disorder to pin the quantized lines so that they are not free to cross the current, permitting resistanceless conduction up to higher values of electric current $[13,14]$. It might be thought that vortices cannot be so easily "pinned" in a classical fluid with smoothly distributed vorticity, but, in fact, the Josephson-Anderson relation (3.50) tells us that any mechanism which reduces drag must somehow decrease vorticity flux across the potential mass current. This includes mechanisms such as drag reduction by polymer additives or the Toms effect $[114,115]$, whose efficacy is well documented but whose detailed physical explanation is still debated. Likewise, anything that enhances drag, such as surface roughness, must increase vorticity flux across the 
potential flow. The best analogy is not with superconductors but instead with neutral superfluids, where surface roughness may likewise increase vortex shedding [116] and interactions of quantized vortices with solid surfaces have long been recognized to play a crucial role in determining critical velocities [117-120].

We argue that empirical investigation of the vorticity flux will provide new clues into the underlying mechanisms of reduced and enhanced drag. Although it has long been supposed that drag must be somehow related to boundarylayer vorticity dynamics, such as separating boundary layers, a precise quantitative connection has been lacking. The Josephson-Anderson formula (3.50) provides an exact relation, which allows the vorticity dynamics underlying drag to be fully explored empirically. The two components of the relation (3.50) are now both available. The inviscid potentialflow solutions can be obtained either analytically by potential-flow theory or by long-established numerical methods [121,122]. The other ingredient, Huggins's vorticity-flux tensor (2.3), can be calculated as well in numerical simulations $[95,96]$ but, furthermore, can be measured even in laboratory experiments of complex turbulent flows. Modern particle imaging velocimetry techniques allow reconstruction of the full 3D flow field, including the vorticity field and its gradients [123]. As discussed already in the Introduction, a version of these methods has recently been applied to resolve the detailed flow features around individual roughness elements of scale $1 \mathrm{~mm}$ in a turbulent inner layer [65-67]. In conjunction with such experimental data, our formula (3.50) can fully elucidate the origin of the increased drag. Likewise in drag-reduced flows, e.g., with polymer additives, the changes in the vorticity dynamics that lead to smaller drag can be identified. Understanding the detailed physical mechanism will allow such techniques to be optimized and adapted to new circumstances.

\section{New theoretical questions}

Granted that the detailed Josephson-Anderson relation (3.50) has important implications, it then becomes interesting to explore the full range of its validity. Although bodies of finite extent are most realistic, it should be illuminating to generalize the relation to more idealized geometries such as flow past cylinders and semi-infinite plates. Validity of the relation for compressible flow would greatly widen the range of applications, and we believe that this could be possible, especially for barotropic models where smooth Euler solutions satisfy a Kelvin circulation theorem and potential-flow conditions are preserved in time. Because of the simplicity of its derivation [16,21,22], the connection between vorticity flux and force balance must be quite general. It is possible that detailed relations of the form of Eqs. (2.13) and (3.50) which relate vorticity flux to energy dissipation have also greater generality than just simple fluids. For example, there are some intriguing parallels with forces on bodies in active fluids [124]. The key question here is what would play the role of the inviscid potential Euler solution.

Some of the most urgent theoretical questions raised by the present work are for quantum superfluids. The version of the detailed Josephson-Anderson relation (3.50) that we derive for classical viscous fluids is more comprehensive and exact than existing versions for superfluids. It therefore becomes a pressing issue whether a detailed JosephsonAnderson relation of similar generality to ours can be derived for a mathematical model of a superfluid, such as the Gross-Pitaevskii model. We note that Onsager's "ideal turbulence" theory has recently been extended to superfluid turbulence described by the Gross-Pitaevskii equation $[125,126]$, which leads to the prediction that the inertial range of superfluid turbulence at scales between the forcing scale $L$ and the mean intervortex length $\ell_{\text {int }}$ is described by a dissipative weak solution of the compressible Euler equations. If this Onsager theory for superfluids can be generalized to wall-bounded flow along the lines of recent work [98-102] for classical fluids, then the dissipative anomaly should be related by a Josephson-Anderson relation to vorticity flux from the boundary. We note that forced flows of superfluid ${ }^{4} \mathrm{He}$ in the two-fluid regime through smooth wall pipes and channels at high Reynolds numbers suffer a pressure drop in reasonable agreement with classical friction laws $[127,128]$, and velocity profiles determined from particle imaging velocimetry exhibit a near-wall turbulent boundary layer [129]. The close correspondence with classical wall-bounded turbulence remains to be understood and calls for further understanding of the processes generating quantized vortices at the walls in superfluids ([4], Secs. IV and V) and the role of surface roughness [116-120].

Finally, there should be a connection of the JosephsonAnderson relation with stochastic Lagrangian representation of the vorticity dynamics [130-133], which gives a more precise meaning to the "motion" of vortex lines in a classical fluid. This approach has recently been generalized to solve the Helmholtz equation with the Lighthill vorticity source as Neumann boundary conditions [134,135], but it is not yet clear how to relate the stochastic Lagrangian trajectories to the Huggins vorticity-flux tensor in the flow interior. These stochastic representations are an exact mathematical approach to realize Huggins's suggestion [21] of a probability interpretation of the vorticity field.

\section{ACKNOWLEDGMENTS}

We wish to thank Ping Ao for his insistence to us in 2006 that energy dissipation in classical fluids should be explainable by the Josephson-Anderson relation, which led us to discover the works of Huggins. We thank also the Simons Foundation for support of this work through the Targeted 
Grant No. MPS-663054, "Revisiting the Turbulence Problem Using Statistical Mechanics."

\section{APPENDIX A: DERIVATION OF HUGGINS'S RELATION}

For completeness, we reproduce here the derivation of the detailed Josephson-Anderson relation for the channelflow geometry originally obtained by Huggins. The starting point is the Bernoulli equation for the scalar potential of the ideal flow:

$$
\dot{\phi}+\frac{1}{2}\left|\mathbf{u}_{\phi}\right|^{2}+p_{\phi}+U=0 .
$$

Its space gradient is the Euler equation for $\mathbf{u}_{\phi}=\boldsymbol{\nabla} \phi$, which, subtracted from the Navier-Stokes equation (2.1), yields the equation of motion for $\mathbf{u}_{\omega}:=\mathbf{u}-\mathbf{u}_{\phi}$ :

$\partial_{t} \mathbf{u}_{\omega}=\mathbf{u} \times \boldsymbol{\omega}-\nu \boldsymbol{\nabla} \times \boldsymbol{\omega}-\mathbf{g}-\boldsymbol{\nabla}\left(p_{\omega}+\frac{1}{2}\left|\mathbf{u}_{\omega}\right|^{2}+\mathbf{u}_{\omega} \cdot \mathbf{u}_{\phi}\right)$,

with the boundary conditions

$\left.\mathbf{n} \cdot \mathbf{u}_{\omega}\right|_{S_{\text {in }}}=\mathbf{0},\left.\quad \mathbf{n} \cdot \mathbf{u}_{\omega}\right|_{S_{\text {out }}}=\mathbf{0},\left.\quad \mathbf{n} \cdot \mathbf{u}_{\omega}\right|_{S_{w}}=\mathbf{0}$.

Dotting Eq. (A2) with $\mathbf{u}_{\omega}$ and integrating over the channel domain $\Omega$ immediately yields

$$
\frac{d E_{\omega}}{d t}=\mathcal{T}-\int_{\Omega}\left[\eta|\boldsymbol{\omega}|^{2}+\rho \mathbf{u} \cdot \mathbf{g}\right] d V
$$

which is Eq. (2.11) in the main text. On the other hand, the total kinetic energy $E(t)=(1 / 2) \int \rho|\mathbf{u}|^{2} d V$ satisfies

$$
\frac{d E}{d t}=\int d J\left(h_{\text {in }}-h_{\text {out }}\right)-\int_{\Omega}\left[\eta|\boldsymbol{\omega}|^{2}+\rho \mathbf{u} \cdot \mathbf{g}\right] d V,
$$

and since $E=E_{\phi}+E_{\omega}$ by the Kelvin minimum-energy theorem, we obtain

$$
\frac{d E_{\phi}}{d t}=\int d J\left(h_{\text {in }}-h_{\text {out }}\right)-\mathcal{T},
$$

which is Eq. (2.10) in the main text. Finally,

$$
\begin{aligned}
\frac{d E_{\phi}}{d t} & =\int_{\Omega} \rho \mathbf{u}_{\phi} \cdot \dot{\mathbf{u}}_{\phi} d V \\
& =\int_{\Omega} \rho \boldsymbol{\nabla} \cdot\left(\dot{\phi} \mathbf{u}_{\phi}\right) d V=\int d J\left(\dot{\phi}_{\text {out }}-\dot{\phi}_{\text {in }}\right)
\end{aligned}
$$

by using the divergence theorem and $d J=\rho \mathbf{u}_{\phi} \cdot d \mathbf{A}$. Combining Eqs. (A6) and (A7) yields

$$
\mathcal{T}=\int d J\left(h_{\text {in }}^{\prime}-h_{\text {out }}^{\prime}\right)
$$

which is the detailed Josephson relation (2.13) first derived by Huggins.

\section{APPENDIX B: VORTEX MOMENTUM AND IMPULSE}

We give here another derivation of Eq. (3.37) different from that of Ref. [88]. Using the identity

$$
\mathbf{x} \times \boldsymbol{\omega}=x_{i} \nabla u_{\omega i}-(\mathbf{x} \cdot \boldsymbol{\nabla}) \mathbf{u}_{\omega},
$$

we find after integration by parts that

$$
\begin{aligned}
\int_{\Omega} \mathbf{x} \times \omega d V= & \int_{\Omega}(-1+3) \mathbf{u}_{\omega} d V \\
& -\int_{\partial B} \mathbf{x} \times\left(\hat{\mathbf{n}} \times \mathbf{u}_{\omega}\right) d A \\
& +\lim _{R \rightarrow \infty} \int_{S_{R}} \mathbf{x} \times\left(\hat{\mathbf{r}} \times \mathbf{u}_{\omega}\right) d A,
\end{aligned}
$$

or from the definition of $\mathbf{I}_{\omega}$,

$$
2 \mathbf{I}_{\omega}=2 \int_{\Omega} \mathbf{u}_{\omega} d V+\lim _{R \rightarrow \infty} \int_{S_{R}} \mathbf{x} \times\left(\hat{\mathbf{r}} \times \mathbf{u}_{\omega}\right) d A .
$$

Using the asymptotic far-field expansion of $\mathbf{u}_{\omega}$,

$$
\begin{aligned}
& \lim _{R \rightarrow \infty} \int_{S_{R}} \mathbf{x} \times\left(\hat{\mathbf{r}} \times \mathbf{u}_{\omega}\right) d A \\
& =\lim _{R \rightarrow \infty} \int_{S_{R}} R \hat{\mathbf{r}} \times \frac{\hat{\mathbf{r}} \times\left(-\mathbf{I}_{\omega}\right)}{4 \pi R^{3}} R^{2} d \Omega \\
& =\frac{1}{4 \pi} \int\left[\mathbf{I}_{\omega}-\left(\mathbf{I}_{\omega} \cdot \hat{\mathbf{r}}\right) \hat{\mathbf{r}}\right] d \Omega=\frac{2}{3} \mathbf{I}_{\omega} .
\end{aligned}
$$

Thus, we finally obtain

$$
\int_{\Omega} \mathbf{u}_{\omega} d V=\frac{2}{3} \mathbf{I}_{\omega}
$$

[1] B. D. Josephson, Possible New Effects in Superconductive Tunnelling, Phys. Lett. 1, 251 (1962).

[2] P. W. Anderson, Considerations on the Flow of Superfluid Helium, Rev. Mod. Phys. 38, 298 (1966).

[3] R. E. Packard, The Role of the Josephson-Anderson Equation in Superfluid Helium, Rev. Mod. Phys. 70, 641 (1998). 
[4] E. Varoquaux, Anderson's Considerations on the Flow of Superfluid Helium: Some Offshoots, Rev. Mod. Phys. 87, 803 (2015).

[5] L. Onsager, Discussion Remark on "The Two Fluid Model for Helium II," by C. J. Gorter, Nuovo Cimento Suppl. 6, 249 (1949).

[6] R. P. Feynman, in Progress in Low Temperature Physics (Elsevier, New York, 1955), Vol. 1, Chap. II, pp. 17-53.

[7] In his original paper, Anderson wrote this equation in a form without the explicit vortex term, valid only away from the vortex line. See Eq. (15) of Ref. [2]. The contemporary version of the relation in Eq. (1.2) (e.g., see Ref. [8]) makes more clear the role of vortex motions.

[8] P. Ao, Spectral Flow, the Magnus Force, and the Josephson-Anderson Relation, Phys. Lett. A 216, 167 (1996).

[9] B. D. Josephson, Potential Differences in the Mixed State of Type II Superconductors, Phys. Lett. 16, 242 (1965).

[10] E. R. Huggins, Energy-Dissipation Theorem and Detailed Josephson Equation for Ideal Incompressible Fluids, Phys. Rev. A 1, 332 (1970).

[11] W. Zimmermann, Energy Transfer and Phase Slip by Quantum Vortex Motion in Superfluid ${ }^{4} \mathrm{He}$, J. Low Temp. Phys. 93, 1003 (1993).

[12] M. Greiter, Is Electromagnetic Gauge Invariance Spontaneously Violated in Superconductors?, Ann. Phys. (Amsterdam) 319, 217 (2005).

[13] D. J. Bishop, P. L. Gammel, and D. A. Huse, Resistance in High-Temperature Superconductors, Sci. Am. 268, 48 (1993).

[14] G. Blatter, M. V. Feigel'man, V. B. Geshkenbein, A. I. Larkin, and V. M. Vinokur, Vortices in High-Temperature Superconductors, Rev. Mod. Phys. 66, 1125 (1994).

[15] The author had a conversation with Anderson about this issue during a sabbatical at Princeton in 2017, and Anderson still maintained the opinion at that time that the relation was of special importance in superfluids where vortices are quantized and was not obviously useful in classical hydrodynamics.

[16] E. R. Huggins, Vortex Currents in Turbulent Superfluid and Classical Fluid Channel Flow, the Magnus Effect, and Goldstone Boson Fields, J. Low Temp. Phys. 96, 317 (1994).

[17] Elisha Huggins was a Ph.D. student of Richard Feynman, from whom he may have inherited his interest in classical fluid turbulence.

[18] G. I. Taylor, The Transport of Vorticity and Heat through Fluids in Turbulent Motion, Proc. R. Soc. A 135, 685 (1932).

[19] M. J. Lighthill, in Laminar Boundary Theory, edited by L. Rosenhead (Oxford University Press, Oxford, 1963), pp. 46-113.

[20] E. R. Huggins, Exact Magnus-Force Formula for ThreeDimensional Fluid-Core Vortices, Phys. Rev. A 1, 327 (1970).

[21] E. R. Huggins, Dynamical Theory and Probability Interpretation of the Vorticity Field, Phys. Rev. Lett. 26, 1291 (1971).

[22] G. L. Eyink, Turbulent Flow in Pipes and Channels as Cross-Stream "Inverse Cascades" of Vorticity, Phys. Fluids 20, 125101 (2008).
[23] L. Kelvin, in Mathematical and Physical Papers: Collected from Different Scientific Periodicals from May, 1841, to the Present Time (Cambridge University Press, Cambridge, England, 1882), Vol. I.

[24] J. le Rond d'Alembert, Theoria resistentiae quam patitur corpus in fluido motum, ex principiis omnino novis et simplissimis deducta, habita ratione tum velocitatis, igurae, et massae corporis moti, tum densitatis compressionis partium fluidi, manuscript at Berlin-Brandenburgische Akademie der Wissenschaften, Akademie-Archiv Call No. I-M478 (1749).

[25] J. le Rond d'Alembert, in Opuscules Mathématiques (Briasson, Paris, 1768), Vol. 5, Sec. I, pp. 132-138.

[26] G. Grimberg, W. Pauls, and U. Frisch, Genesis of d'Alembert's Paradox and Analytical Elaboration of the Drag Problem, Physica (Amsterdam) 237D, 1878 (2008).

[27] T. Frisch, Y. Pomeau, and S. Rica, Transition to Dissipation in a Model of Superflow, Phys. Rev. Lett. 69, 1644 (1992).

[28] B. Jackson, J. F. McCann, and C. S. Adams, Vortex Formation in Dilute Inhomogeneous Bose-Einstein Condensates, Phys. Rev. Lett. 80, 3903 (1998).

[29] T. Winiecki, J. F. McCann, and C. S. Adams, Pressure Drag in Linear and Nonlinear Quantum Fluids, Phys. Rev. Lett. 82, 5186 (1999).

[30] T. Winiecki, B. Jackson, J. F. McCann, and C. S. Adams, Vortex Shedding and Drag in Dilute Bose-Einstein Condensates, J. Phys. B 33, 4069 (2000).

[31] T. Winiecki and C. S. Adams, Motion of an Object through a Quantum Fluid, Europhys. Lett. 52, 257 (2000).

[32] T. Winiecki, Numerical Studies of Superfluids and Superconductors, Ph.D. thesis, Durham University, 2001.

[33] C. Huepe and M.-E. Brachet, Scaling Laws for Vortical Nucleation Solutions in a Model of Superflow, Physica (Amsterdam) 140D, 126 (2000).

[34] C. Nore, C. Huepe, and M.E. Brachet, Subcritical Dissipation in Three-Dimensional Superflows, Phys. Rev. Lett. 84, 2191 (2000).

[35] C.-T. Pham, C. Nore, and M.-É. Brachet, Boundary Layers and Emitted Excitations in Nonlinear Schrödinger Superflow Past a Disk, Physica (Amsterdam) 210D, 203 (2005).

[36] K. Sasaki, N. Suzuki, and H. Saito, Bénard-von Kármán Vortex Street in a Bose-Einstein Condensate, Phys. Rev. Lett. 104, 150404 (2010).

[37] G. W. Stagg, N. G. Parker, and C. F. Barenghi, Quantum Analogues of Classical Wakes in Bose-Einstein Condensates, J. Phys. B 47, 095304 (2014).

[38] G. W. Stagg, A. J. Allen, N. G. Parker, and C. F. Barenghi, Generation and Decay of Two-Dimensional Quantum Turbulence in a Trapped Bose-Einstein Condensate, Phys. Rev. A 91, 013612 (2015).

[39] S. Musser, D. Proment, M. Onorato, and W. T. M. Irvine, Starting Flow Past an Airfoil and Its Acquired Lift in a Superfluid, Phys. Rev. Lett. 123, 154502 (2019).

[40] L. Onsager, Statistical Hydrodynamics, Nuovo Cimento Suppl. 6, 279 (1949).

[41] G. L. Eyink and K. R. Sreenivasan, Onsager and the Theory of Hydrodynamic Turbulence, Rev. Mod. Phys. 78, 87 (2006). 
[42] G. L. Eyink, Review of the Onsager, Ideal Turbulence Theory, arXiv:1803.02223.

[43] A. Saint-Venant, Résistance des fluides: considérations historiques, physiques et pratiques relatives au problème de l'action dynamique muteulle d'un fluide a d'un solide, dans l'état de permanence supposé acquis par leurs mouvements, Acad. Sci. Mém. 44, 1 (1846).

[44] L. Prandtl, in Proceedings of the Third International Congress of Mathematics, Heidelberg, 1904, https://ntrs .nasa.gov/api/citations/19930090813/downloads/ 19930090813.pdf.

[45] K. Stewartson, D'Alembert's Paradox, SIAM Rev. 23, 308 (1981).

[46] E. Achenbach, Experiments on the Flow Past Spheres at Very High Reynolds Numbers, J. Fluid Mech. 54, 565 (1972).

[47] T. D. Drivas and H. Q. Nguyen, Remarks on the Emergence of Weak Euler Solutions in the Vanishing Viscosity Limit, J. Nonlinear Sci. 29, 709 (2019).

[48] O. Cadot, Y. Couder, A. Daerr, S. Douady, and A. Tsinober, Energy Injection in Closed Turbulent Flows: Stirring through Boundary Layers versus Inertial Stirring, Phys. Rev. E 56, 427 (1997).

[49] J. Nikuradse, Gesetzmässigkeitem der turbulentem Strömung in glatten Rohren, VD-Forschungsheft 356, 136 (1932) [Laws of Turbulent Flow in Smooth Pipes (1966), https://archive.org/details/nasa_techdoc_19670004508].

[50] B. J. McKeon, C. J. Swanson, M. V. Zagarola, R. J. Donnelly, and A. J. Smits, Friction Factors for Smooth Pipe Flow, J. Fluid Mech. 511, 41 (2004).

[51] R. Örlü, T. Fiorini, A. Segalini, G. Bellani, A. Talamelli, and P. H. Alfredsson, Reynolds Stress Scaling in Pipe Flow Turbulence-First Results from CICLoPE, Phil. Trans. R. Soc. A 375, 20160187 (2017).

[52] N. Furuichi, Y. Terao, Y. Wada, and Y. Tsuji, Further Experiments for Mean Velocity Profile of Pipe Flow at High Reynolds Number, Phys. Fluids 30, 055101 (2018).

[53] J. J. Niemela, L. Skrbek, K. R. Sreenivasan, and R. J. Donnelly, Turbulent Convection at Very High Rayleigh Numbers, Nature (London) 404, 837 (2000).

[54] P.-E. Roche, F. Gauthier, R. Kaiser, and J. Salort, On the Triggering of the Ultimate Regime of Convection, New J. Phys. 12, 085014 (2010).

[55] G. Ahlers, E. Bodenschatz, and X. He, Ultimate-State Transition of Turbulent Rayleigh-Bénard Convection, Phys. Rev. Fluids 2, 054603 (2017).

[56] J. Nikuradse, Strömungsgesetze in rauhen Rohren, VDIForschungsheft 361, 1 (1933); [Laws of Flow in Rough Pipes (National Advisory Committee for Aeronautics, Washington, DC, 1950), https://digital.library.unt.edu/ark \%3A/67531/metadc63009/m2/1/high_res_d/ 19930093938.pdf].

[57] M. A. Shockling, J. J. Allen, and A. J. Smits, Roughness Effects in Turbulent Pipe Flow, J. Fluid Mech. 564, 267 (2006).

[58] J. J. Allen, M. A. Shockling, G. J. Kunkel, and A. J. Smits, Turbulent Flow in Smooth and Rough Pipes, Phil. Trans. R. Soc. A 365, 699 (2007).

[59] X. Zhu, R. J. A. M. Stevens, O. Shishkina, R. Verzicco, and De. Lohse, $N u \sim R a^{1 / 2}$ Scaling Enabled by Multiscale
Wall Roughness in Rayleigh-Bénard Turbulence, J. Fluid Mech. 869, R4 (2019).

[60] K. R. Sreenivasan, On the Scaling of the Turbulence Energy Dissipation Rate, Phys. Fluids 27, 1048 (1984).

[61] B. R. Pearson, P.-A. Krogstad, and W. van de Water, Measurements of the Turbulent Energy Dissipation Rate, Phys. Fluids 14, 1288 (2002).

[62] L. Djenidi, N. Lefeuvre, M. D. Kamruzzaman, and R. A. Antonia, On the Normalized Dissipation Parameter in Decaying Turbulence, J. Fluid Mech. 817, 61 (2017).

[63] A. Roshko, Experiments on the Flow Past a Circular Cylinder at Very High Reynolds Number, J. Fluid Mech. 10, 345 (1961).

[64] J. Choi, W.-P. Jeon, and H. Choi, Mechanism of Drag Reduction by Dimples on a Sphere, Phys. Fluids 18, 041702 (2006).

[65] J. Gao and J. Katz, Self-Calibrated Microscopic DualView Tomographic Holography for 3D Flow Measurements, Opt. Express 26, 16708 (2018).

[66] J. Gao and J. Katz, Three-Dimensional Flow Structures and Their Interactions around a Pair of Cubic Roughness Elements Embedded in the Inner Part of a Turbulent Channel Flow, Bull. Am. Phys. Soc. (2020), https:// meetings.aps.org/Meeting/DFD20/Session/Q05.8.

[67] J. Gao, K. Agarwal, and J. Katz, Experimental Investigation of the 3D Flow Structure around a Pair of Cubes Immersed in the Inner Part of a Turbulent Channel Flow, J. Fluid Mech. 918, A31 (2021).

[68] H. Lamb, Hydrodynamics, Dover Books on Physics (Dover Publications, New York, 1945).

[69] G. K. Batchelor, An Introduction to Fluid Dynamics, Cambridge Mathematical Library (Cambridge University Press, Cambridge, England 2000).

[70] J. Z. Wu, H. Ma, and M. D. Zhou, Vorticity and Vortex Dynamics, Lecture Notes in Mathematics (Springer, Berlin, 2007).

[71] L. D. Landau, The Theory of Superfluidity of Helium II, Zh. Eksp. Teor. Fiz. 11, 592 (1941) [J. Phys. U.S.S.R 5, 71 (1941)].

[72] F. A. Lyman, Vorticity Production at a Solid Boundary, Appl. Mech. Rev. 43, 157 (1990) in L. M. Trefethen and R. L. Panton, Some Unanswered Questions in Fluid Mechanics, Appl. Mech. Rev. 43, 153 (1990).

[73] R. L. Panton, Incompressible Flow (John Wiley \& Sons, New York, 1984).

[74] J.-Z. Wu and J.-M. Wu, Interactions between a Solid Surface and a Viscous Compressible Flow Field, J. Fluid Mech. 254, 183 (1993).

[75] J. Z. Wu and J. M. Wu, Vorticity Dynamics on Boundaries, Adv. Appl. Mech. 32, 119 (1996).

[76] J. Z. Wu and J. M. Wu, Boundary Vorticity Dynamics since Lighthill's 1963 Article: Review and Development, Theor. Comput. Fluid Dyn. 10, 459 (1998).

[77] B. R. Morton, The Generation and Decay of Vorticity, Geophys. Astrophys. Fluid Dyn. 28, 277 (1984).

[78] A. Kustepeli, On the Helmholtz Theorem and Its Generalization for Multi-Layers, Electromagnetics 36, 135 (2016).

[79] V. A. Vladimirov, Vortical Momentum of Flows of an Incompressible Liquid (in Russian), Zh. Prikl. Mekh. Tekh. 
Fiz. 18, 72 (1977) [J. Appl. Mech. Tech. Phys. 18, 791 (1978)].

[80] R. B. Payne, Aeronautical Research Council Reports and Memoranda No. 3047 (Her Majesty's Stationary Office, London, 1956), pp. 1-50.

[81] R. B. Payne, Calculations of Unsteady Viscous Flow Past a Circular Cylinder, J. Fluid Mech. 4, 81 (1958).

[82] P. Ploumhans, G. S. Winckelmans, J. K. Salmon, A. Leonard, and M.S. Warren, Vortex Methods for Direct Numerical Simulation of Three-Dimensional Bluff Body Flows: Application to the Sphere at $\mathrm{Re}=300,500$, and 1000, J. Comput. Phys. 178, 427 (2002).

[83] E. Branlard, in Wind Turbine Aerodynamics and VorticityBased Methods, Research Topics in Wind Energy Vol. 7 (Springer, New York, 2017), Chap. 41, pp. 493-543.

[84] K. W. Schwarz, Three-Dimensional Vortex Dynamics in Superfluid ${ }^{4} \mathrm{He}:$ Line-Line and Line-Boundary Interactions, Phys. Rev. B 31, 5782 (1985).

[85] A. Föppl, Die Geometrie der Wirbelfelder (Teubner, Leipzig, 1897).

[86] M. W. Hirsch, Differential Topology, Graduate Texts in Mathematics (Springer, New York, 2012).

[87] H. Federer, Geometric Measure Theory, Classics in Mathematics (Springer, Berlin, 2014).

[88] B. J. Cantwell, Viscous Starting Jets, J. Fluid Mech. 173, 159 (1986).

[89] K. W. Schwarz, Onset of Phase Slip in Superflow through Channels, Phys. Rev. B 12, 3658 (1975).

[90] Note that Refs. [4,89] and the superfluid community in general use the notation $\mathbf{P}$ for the vortex impulse $\rho \mathbf{I}$.

[91] Notice that, until this point, none of our analysis requires that the velocity at infinity $\mathbf{V}(t)$ must be constant in time, and so it applies to more general cases of a body linearly accelerating through a fluid, but without body rotation. The Bernoulli equation (3.6) simply requires a new term $\dot{\mathbf{V}}(t) \cdot \mathbf{x}$ on the right-hand side to obtain the Euler equation in the noninertial body frame. Body rotation, on the other hand, leads to new effects that are beyond the scope of the present paper.

[92] It is important to note that all of the spatial integrals over $\Omega$ are absolutely convergent because of the decay laws $\mathbf{u}_{\omega} \times \boldsymbol{\omega} \sim r^{-7}, \boldsymbol{\nabla} \times \boldsymbol{\omega} \sim r^{-5}$, and $|\boldsymbol{\omega}|^{2} \sim r^{-8}$ asymptotically at large $r$. Thus, the integrands both in the detailed Josephson-Anderson relation (3.50) and in the global viscous dissipation in Eq. (3.49) are well localized, and the integrals can be calculated accurately in a possibly large but finite-volume region of space.

[93] Note that the two integrands in the second line of Eq. (3.55) are separately only conditionally convergent, but their combination, which equals the integrand in the first line, is absolutely convergent.

[94] S. S. Tiwari, E. Pal, S. Bale, N. Minocha, A. W Patwardhan, K. Nandakumar, and J. B. Joshi, Flow Past a Single Stationary Sphere, 2. Regime Mapping and Effect of External Disturbances, Powder Technol. 365, 215 (2020).

[95] G. L. Brown and A. Roshko, Turbulent Shear Layers and Wakes, J. Turbul. N 13, 51 (2012).

[96] S. J. Terrington, K. Hourigan, and M. C. Thompson, The Generation and Diffusion of Vorticity in
Three-Dimensional Flows: Lyman's Flux, J. Fluid Mech. 915, A106 (2021).

[97] Note that the authors of Ref. [96] refer to Huggins's flux in Eq. (2.3) as "Lyman-Huggins flux," citing also Ref. [72]. This double attribution is not really proper, in our opinion, since Lyman considered vorticity generated only at the boundary, whereas it was Huggins who first considered this flux in the interior of the flow.

[98] C. Bardos and E. S. Titi, Onsager's Conjecture for the Incompressible Euler Equations in Bounded Domains, Arch. Ration. Mech. Anal. 228, 197 (2018).

[99] T. D. Drivas and H. Q. Nguyen, Onsager's Conjecture and Anomalous Dissipation on Domains with Boundary, SIAM J. Math. Anal. 50, 4785 (2018).

[100] C. Bardos, E. S. Titi, and E. Wiedemann, Onsager's Conjecture with Physical Boundaries and an Application to the Vanishing Viscosity Limit, Commun. Math. Phys. 370, 291 (2019).

[101] R. M. Chen, Z. Liang, and D. Wang, A Kato-Type Criterion for Vanishing Viscosity near the Onsager's Critical Regularity, arXiv:2007.12746.

[102] G. Eyink, S. Kumar, and H. Quan, The Onsager Theory of Wall-Bounded Turbulence and Taylor's Momentum Anomaly, Philos. Trans. R. Soc. A (to be published).

[103] J. Duchon and R. Robert, Inertial Energy Dissipation for Weak Solutions of Incompressible Euler and Navier-Stokes Equations, Nonlinearity 13, 249 (2000).

[104] G. L. Eyink, H. Quan, and S. Kumar, Onsager's Theory of Turbulence and the Modern D'Alembert Paradox (to be published).

[105] U. Piomelli, Recent Advances in the Numerical Simulation of Rough-Wall Boundary Layers, Phys. Chem. Earth 113, 63 (2019).

[106] We can use the total pressure $P$ here rather than the rotational contribution $P_{\omega}$ because the form drag from the potential -flow pressure $P_{\phi}$ is zero by d'Alembert's theorem $[24,25]$.

[107] A. Busse, M. Thakkar, and N. D. Sandham, ReynoldsNumber Dependence of the Near-Wall Flow over Irregular Rough Surfaces, J. Fluid Mech. 810, 196 (2017).

[108] J. Jiménez, Turbulent Flows over Rough Walls, Annu. Rev. Fluid Mech. 36, 173 (2004).

[109] The only countervailing data of which we are aware are that plotted in Fig. 2 of Ref. [48]. For the smooth-wall von Kármán flow, a surrogate measure of interior dissipation constructed from pressure fluctuations showed apparent $\mathrm{Re}$ independence for $\operatorname{Re}=10^{4}-10^{6}$. If this behavior were to persist up to $\operatorname{Re}=10^{8}-10^{9}$, then as noted in Ref. [48], the Re-independent energy dissipation in the bulk would surpass the declining dissipation in the near-wall boundary layer. However, this data is not a direct observation of anomalous dissipation and requires bold extrapolations to conclude such an anomaly.

[110] G. Luo and T. Y. Hou, Potentially Singular Solutions of the 3D Axisymmetric Euler Equations, Proc. Natl. Acad. Sci. U.S.A. 111, 12968 (2014).

[111] G. Luo and T. Y. Hou, Formation of Finite-Time Singularities in the $3 D$ Axisymmetric Euler Equations: A Numerics Guided Study, SIAM Rev. 61, 793 (2019). 
[112] K. R. Sreenivasan, An Update on the Energy Dissipation Rate in Isotropic Turbulence, Phys. Fluids 10, 528 (1998).

[113] Y. Kaneda, T. Ishihara, M. Yokokawa, K. Itakura, and A. Uno, Energy Dissipation Rate and Energy Spectrum in High Resolution Direct Numerical Simulations of Turbulence in a Periodic Box, Phys. Fluids 15, L21 (2003).

[114] B. A. Toms, Some Observations on the Flow of Linear Polymer Solutions through Straight Tubes at Large Reynolds Numbers, Proc. Intl. Congr. Rheol. 2, 135 (1948).

[115] C. M. White and M. G. Mungal, Mechanics and Prediction of Turbulent Drag Reduction with Polymer Additives, Annu. Rev. Fluid Mech. 40, 235 (2008).

[116] G. W. Stagg, N. G. Parker, and C. F. Barenghi, Superfluid Boundary Layer, Phys. Rev. Lett. 118, 135301 (2017).

[117] S. G. Hegde and W. I. Glaberson, Pinning of Superfluid Vortices to Surfaces, Phys. Rev. Lett. 45, 190 (1980).

[118] K. W. Schwarz, Vortex Pinning in Superfluid Helium, Phys. Rev. Lett. 47, 251 (1981).

[119] K. W. Schwarz, Effect of Surface Roughness on the Critical Velocities of Superfluid ${ }^{4} \mathrm{He}$, Phys. Rev. Lett. 69, 3342 (1992).

[120] P. Hakonen, O. Avenel, and E. Varoquaux, Evidence for Single-Vortex Pinning and Unpinning Events in Superfluid ${ }^{4}$ He, Phys. Rev. Lett. 81, 3451 (1998).

[121] J. L. Hess and A. M. O. Smith, Calculation of Potential Flow about Arbitrary Bodies, Prog. Aerosp. Sci. 8, 1 (1967).

[122] E. F. Kaasschieter and A. J. M. Huijben, Mixed-Hybrid Finite Elements and Streamline Computation for the Potential Flow Problem, Numer. Methods Partial Differ. Equ. 8, 221 (1992).

[123] J. Westerweel, G. E. Elsinga, and R. J. Adrian, Particle Image Velocimetry for Complex and Turbulent Flows, Annu. Rev. Fluid Mech. 45, 409 (2013).

[124] T. Speck and A. Jayaram, Vorticity Determines the Force on Bodies Immersed in Active Fluids, Phys. Rev. Lett. 126, 138002 (2021).
[125] T. Dębiec, P. Gwiazda, A. Świerczewska-Gwiazda, and A. Tzavaras, Conservation of Energy for the Euler-Korteweg Equations, Calc. Var. Partial Differ. Equ. 57, 1 (2018).

[126] T. Tanogami, Theoretical Analysis of Quantum Turbulence Using the Onsager Ideal Turbulence Theory, Phys. Rev. E 103, 023106 (2021).

[127] C. J. Swanson, R. J. Donnelly, and G. G. Ihas, Turbulent Pipe Flow of He I and He II, Physica (Amsterdam) 284B, 77 (2000).

[128] S. Fuzier, B. Baudouy, and S. W. Van Sciver, SteadyState Pressure Drop and Heat Transfer in He II Forced Flow at High Reynolds Number, Cryogenics 41, 453 (2001).

[129] T. Xu and S. W. Van Sciver, Particle Image Velocimetry Measurements of the Velocity Profile in He II Forced Flow, Phys. Fluids 19, 071703 (2007).

[130] P. Constantin and G. Iyer, A Stochastic Lagrangian Representation of the Three-Dimensional Incompressible Navier-Stokes Equations, Commun. Pure Appl. Math. 61, 330 (2008).

[131] P. Constantin and G. Iyer, A Stochastic-Lagrangian Approach to the Navier-Stokes Equations in Domains with Boundary, Ann. Appl. Probab. 21, 1466 (2011).

[132] G. L. Eyink, A. Gupta, and T. A. Zaki, Stochastic Lagrangian Dynamics of Vorticity. Part 1. General Theory for Viscous, Incompressible Fluids, J. Fluid Mech. 901, A2 (2020).

[133] G. L. Eyink, A. Gupta, and T. A. Zaki, Stochastic Lagrangian Dynamics of Vorticity. Part 2. Application to NearWall Channel-Flow Turbulence, J. Fluid Mech. 901, A3 (2020).

[134] G. L. Eyink and L. Peng, Stochastic Lagrangian Dynamics of Vorticity with Neumann Boundary Conditions (to be published).

[135] M. Wang, T. Zaki, and G. L. Eyink, Origin of Enhanced Drag in a Transitional and Zero Pressure-Gradient Boundary Layer (to be published). 